\title{
Bay substituted thiaza[5]helicenes: Synthesis and implications on structural and spectroscopic properties
}

Mathias Daniels,[a] Flip de Jong,[b] Tom Vandermeeren,[a] Luc Van Meervelt,[c] Mark Van der Auweraer,[b] and Wim Dehaen*[a]

[a] Molecular Design and Synthesis, Department of Chemistry, KU Leuven, Leuven Chem\&Tech Celestijnenlaan 200F, B-3001 Leuven, Belgium wim.dehaen@kuleuven.be

[b] Molecular Imaging and Photonics, Department of Chemistry, KU Leuven, Leuven Chem\&Tech Celestijnenlaan 200F, B-3001 Leuven, Belgium.

[c] Biochemistry, Molecular and Structural Biology, Department of Chemistry, KU Leuven, Leuven Chem\&Tech - Celestijnenlaan 200F, B-3001 Leuven, Belgium.

\section{Table of contents}

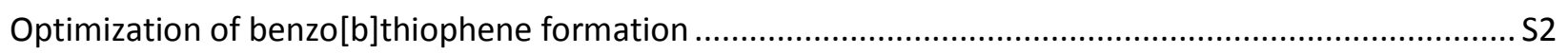

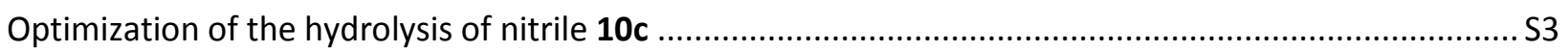

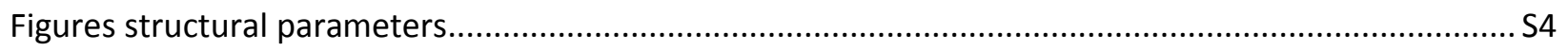

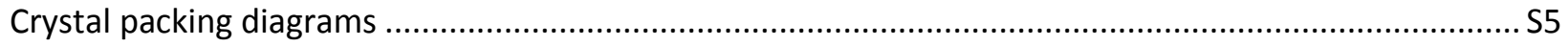

Absorption, emission and excitation spectra ….................................................................................. S12

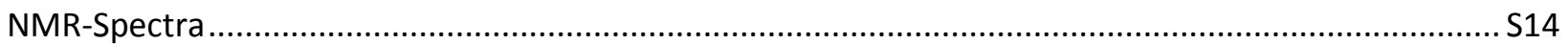




\section{Optimization of benzo[b]thiophene formation}

A selection of the screened reaction conditions for the synthesis of benzo[ $b$ ]thiophene 6 through a base mediated condensation reaction between 2-chlorobenzaldehyde $\mathbf{5}$ and 2-bromobenzylthiol $\mathbf{4}$ can be found in Table S1 (SI). Increasing the temperature further appeared to have only a minor effect (entry 3), while both solvent and base were found to have a significant impact on the isolated yield. We explored more environmentally friendly solvents like glycerol, dimethyl sulfoxide and diglyme, but all gave inferior results compared to dimethylformamide. We found that both cesium carbonate and sodium hydroxide in dimethylformamide gave good yields (entries 6 and 10), while potassium carbonate, even when a larger amount was used or in combination with 18-crown-6, only resulted in moderate yields. On larger scale, the isolated yield of 6 increased, probably due to a decreased exposure to oxygen (entry 11).

Table S1: Optimisation of the reaction conditions for benzo[b]thiophene formation

\begin{tabular}{|c|c|c|c|c|}
\hline Entry & Base/additive & Solvent & Temperature (time) & Yield (\%)a \\
\hline 1 & $\mathrm{~K}_{2} \mathrm{CO}_{3}$ & DMF & $60^{\circ} \mathrm{C}(18 \mathrm{~h})$ then $150^{\circ} \mathrm{C}(23 \mathrm{~h})$ & 36 \\
\hline 2 & $\mathrm{~K}_{2} \mathrm{CO}_{3}$ & DMF & $150^{\circ} \mathrm{C}(41 \mathrm{~h})$ & 50 \\
\hline 3 & $\mathrm{~K}_{2} \mathrm{CO}_{3}$ & DMF & $180^{\circ} \mathrm{C}(41 \mathrm{~h})$ & 55 \\
\hline 4 & $\mathrm{~K}_{2} \mathrm{CO}_{3}$ & DMF & $150^{\circ} \mathrm{C}(41 \mathrm{~h})$ & $53^{b}$ \\
\hline 5 & $\mathrm{~K}_{2} \mathrm{CO}_{3}(18$ crown6) & DMF & $150^{\circ} \mathrm{C}(41 \mathrm{~h})$ & 59 \\
\hline 6 & $\mathrm{NaOH}$ & DMF & $150^{\circ} \mathrm{C}(41 \mathrm{~h})$ & 76 \\
\hline 7 & $\mathrm{NaOt}-\mathrm{Bu}$ & DMF & $150^{\circ} \mathrm{C}(41 \mathrm{~h})$ & n.d. \\
\hline 8 & $\mathrm{NaNH}_{2}$ & DMF & $150^{\circ} \mathrm{C}(41 \mathrm{~h})$ & trace \\
\hline 9 & DBU & DMF & $150^{\circ} \mathrm{C}(41 \mathrm{~h})$ & 44 \\
\hline 10 & $\mathrm{Cs}_{2} \mathrm{CO}_{3}$ & DMF & $150^{\circ} \mathrm{C}(41 \mathrm{~h})$ & 72 \\
\hline 11 & $\mathrm{Cs}_{2} \mathrm{CO}_{3}$ & DMF & $150^{\circ} \mathrm{C}(41 \mathrm{~h})$ & $80^{c}$ \\
\hline 12 & $\mathrm{Cs}_{2} \mathrm{CO}_{3}$ & No solvent & $150^{\circ} \mathrm{C}(41 \mathrm{~h})$ & 20 \\
\hline 13 & $\mathrm{Cs}_{2} \mathrm{CO}_{3}$ & DMSO & $150^{\circ} \mathrm{C}(41 \mathrm{~h})$ & 30 \\
\hline 14 & $\mathrm{Cs}_{2} \mathrm{CO}_{3}$ & DMA & $150^{\circ} \mathrm{C}(41 \mathrm{~h})$ & 61 \\
\hline 15 & $\mathrm{Cs}_{2} \mathrm{CO}_{3}$ & diglyme & $150^{\circ} \mathrm{C}(41 \mathrm{~h})$ & 67 \\
\hline 16 & $\mathrm{Cs}_{2} \mathrm{CO}_{3}$ & glycerol & $150^{\circ} \mathrm{C}(41 \mathrm{~h})$ & 33 \\
\hline
\end{tabular}

Reactions performed with 2-chlorobenzaldehyde ( $0.2 \mathrm{mmol}), 2$-bromobenzylthiol $(0.2 \mathrm{mmol})$, base $(0.4 \mathrm{mmol})$ and additive (when used, $0.04 \mathrm{mmol})$ in DMF $(1 \mathrm{ml})$ a. isolated yield b. $0.8 \mathrm{mmol}$ base used. c. reaction performed on $10 \mathrm{mmol}$ scale. $n . d .:$ not detected 
Optimization of the hydrolysis of nitrile $10 \mathrm{c}$

Table.S2: reaction conditions for the hydrolysis of nitrile $10 \mathrm{c}$

$\begin{array}{cccccr}\text { Entry } & \text { Reagent } & \text { Solvent } & \text { Temperatu } & \text { Duration } & \text { result } \\ 1 & \mathrm{H}_{2} \mathrm{SO}_{4} & \text { water } & 100^{\circ} \mathrm{C} & 10 \text { days } & \text { n.c. } \\ 2 & \mathrm{H}_{2} \mathrm{SO}_{4} & \text { water/ 2-(2-methoxyethoxy)ethanol } & 120^{\circ} \mathrm{C} & 2 \text { days } & \mathbf{1 0 e} \\ 3 & \mathrm{NaOH} & \text { water/ dioxane } & 100^{\circ} \mathrm{C} & 6 \text { days } & \text { n.c. } \\ 4 & \mathrm{NaOH} & \text { 2-methoxyethanol } & 100^{\circ} \mathrm{C} & 6 \text { days } & \mathbf{1 0 e} \\ 5 & \mathrm{NaOH} & \text { ethylene glycol } & 150^{\circ} \mathrm{C} & 6 \text { days } & \text { n.c. } \\ 6 & \mathrm{NaOH} & \text { 2-(2-methoxyethoxy)ethanol } & 150^{\circ} \mathrm{C} & 2 \mathrm{~h} & \mathbf{1 0 e}\end{array}$

Reactions performed on $0.05 \mathrm{mmol}$ scale with $0.5 \mathrm{mmol}$ reagent in $0.5 \mathrm{ml}$ solvent. n.c. no conversion 
Figures structural parameters

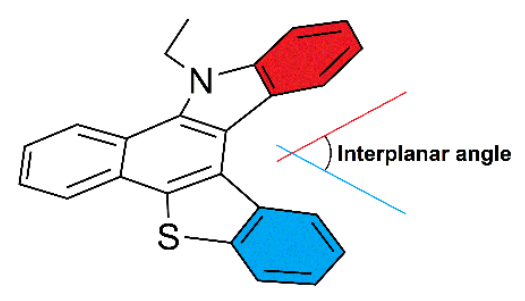

Figure S1: Interplanar angle between the terminal rings of the helicene (red and blue).

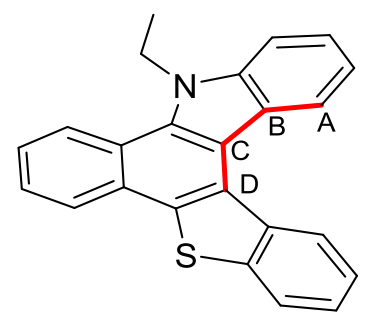

Torsion angle 1

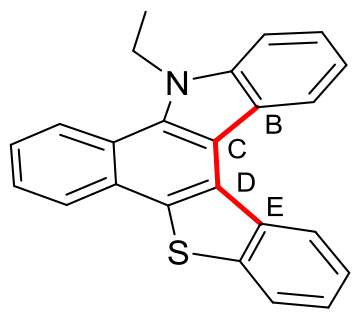

Torsion angle 2

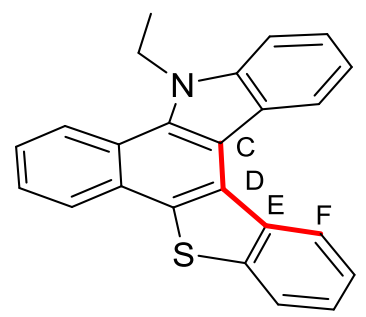

Torsion angle 3

Figure S2: Torsion angles 1-3 between bonds $A B-C D, B C-D E$ and $C D-E F$, respectively.<smiles></smiles>

Figure S3: Distance between the bay area carbon atoms. 
Crystal packing diagrams

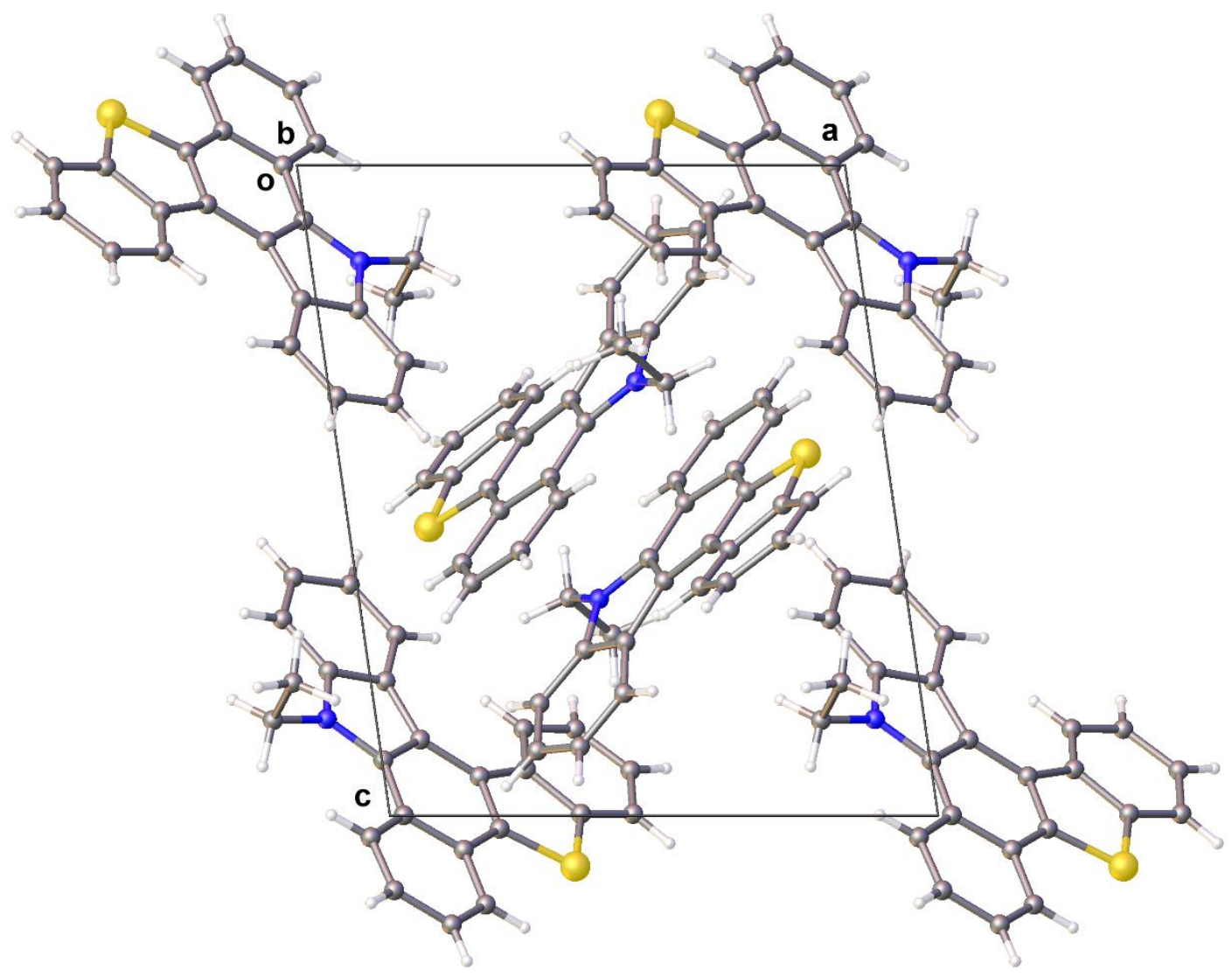

Figure S4: Packing in crystal structure of 10a 


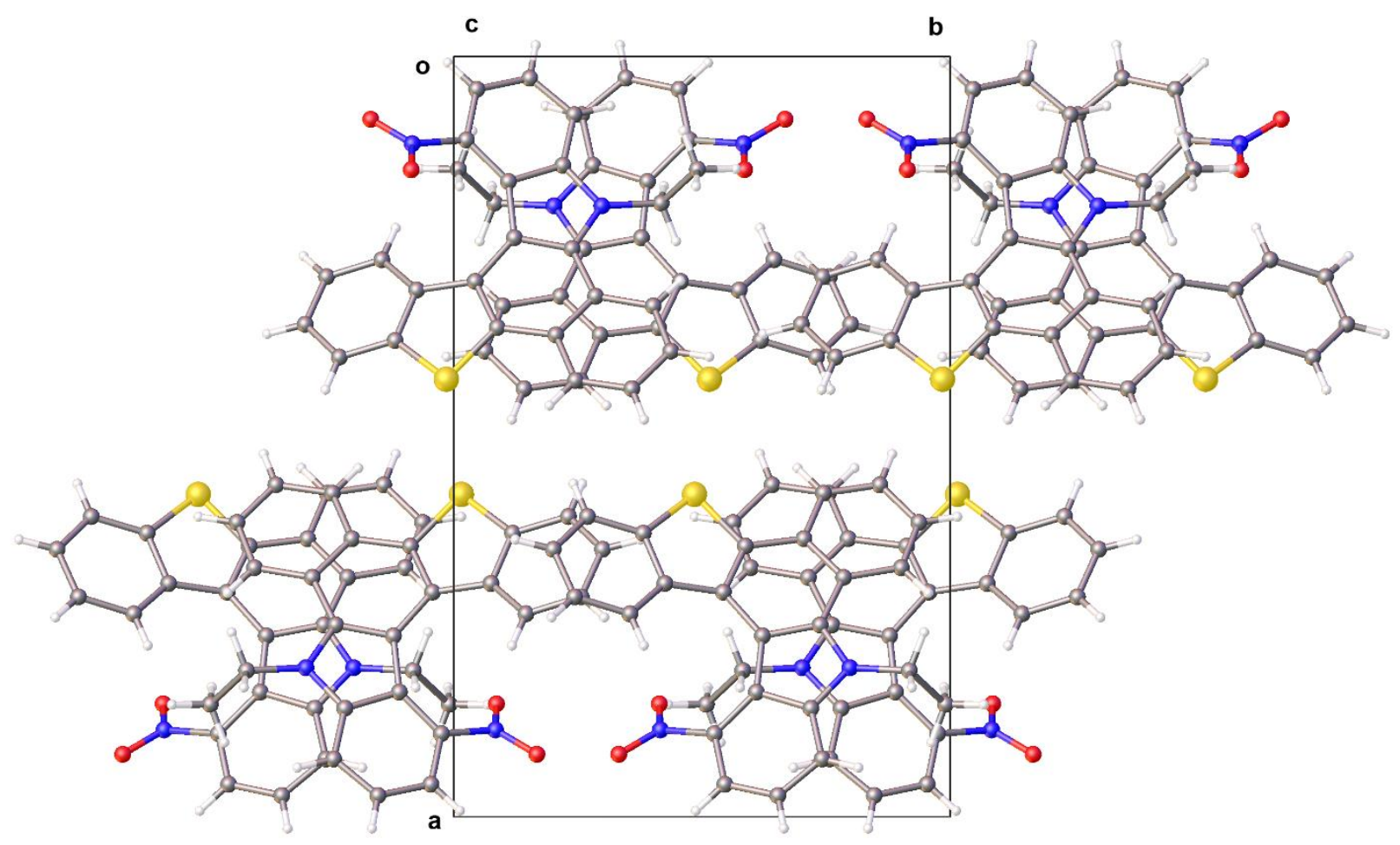

Figure S5: Packing in crystal structure of $\mathbf{1 0 b}$ 


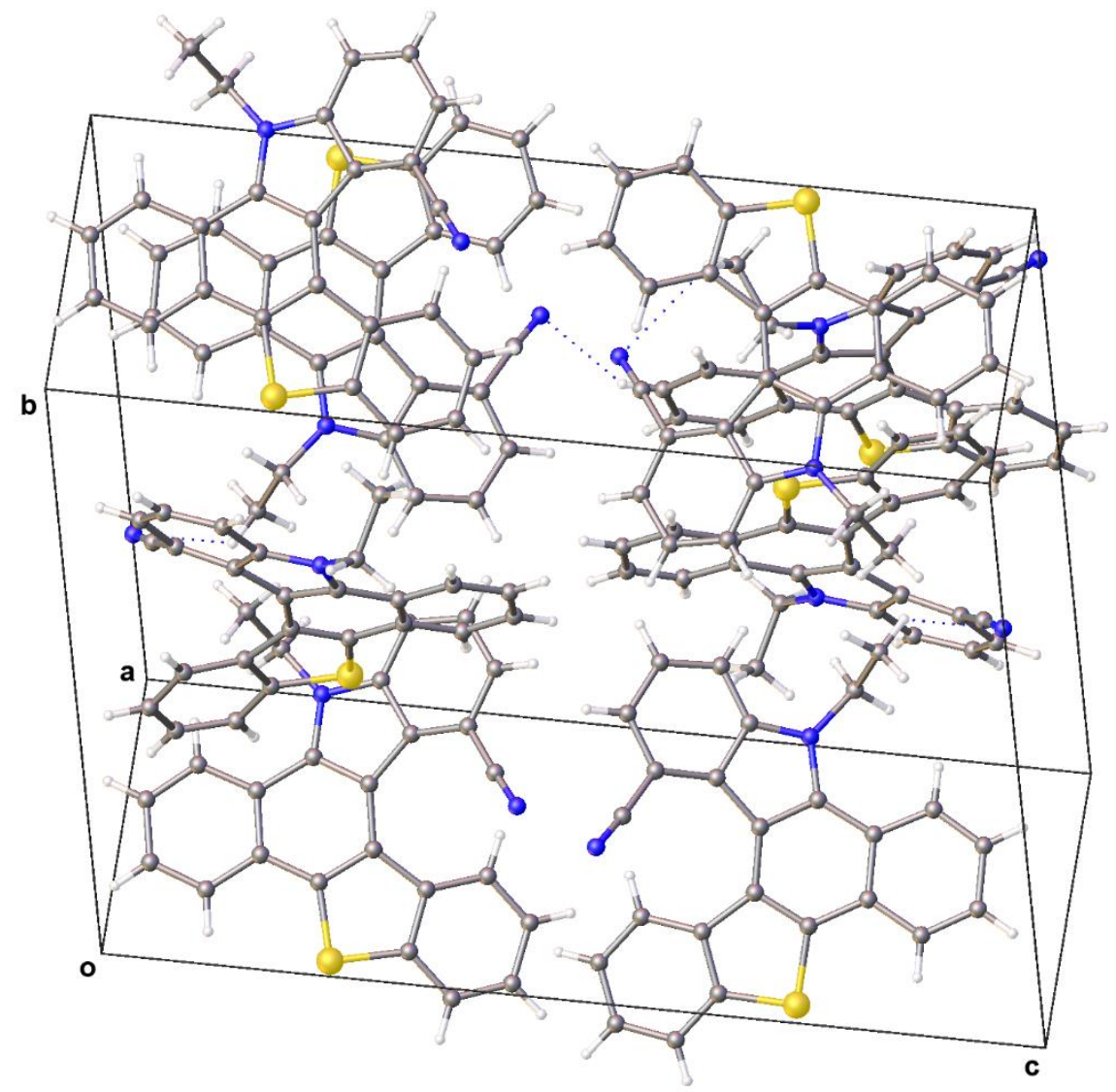

Figure S6: Packing in crystal structure of 10c 


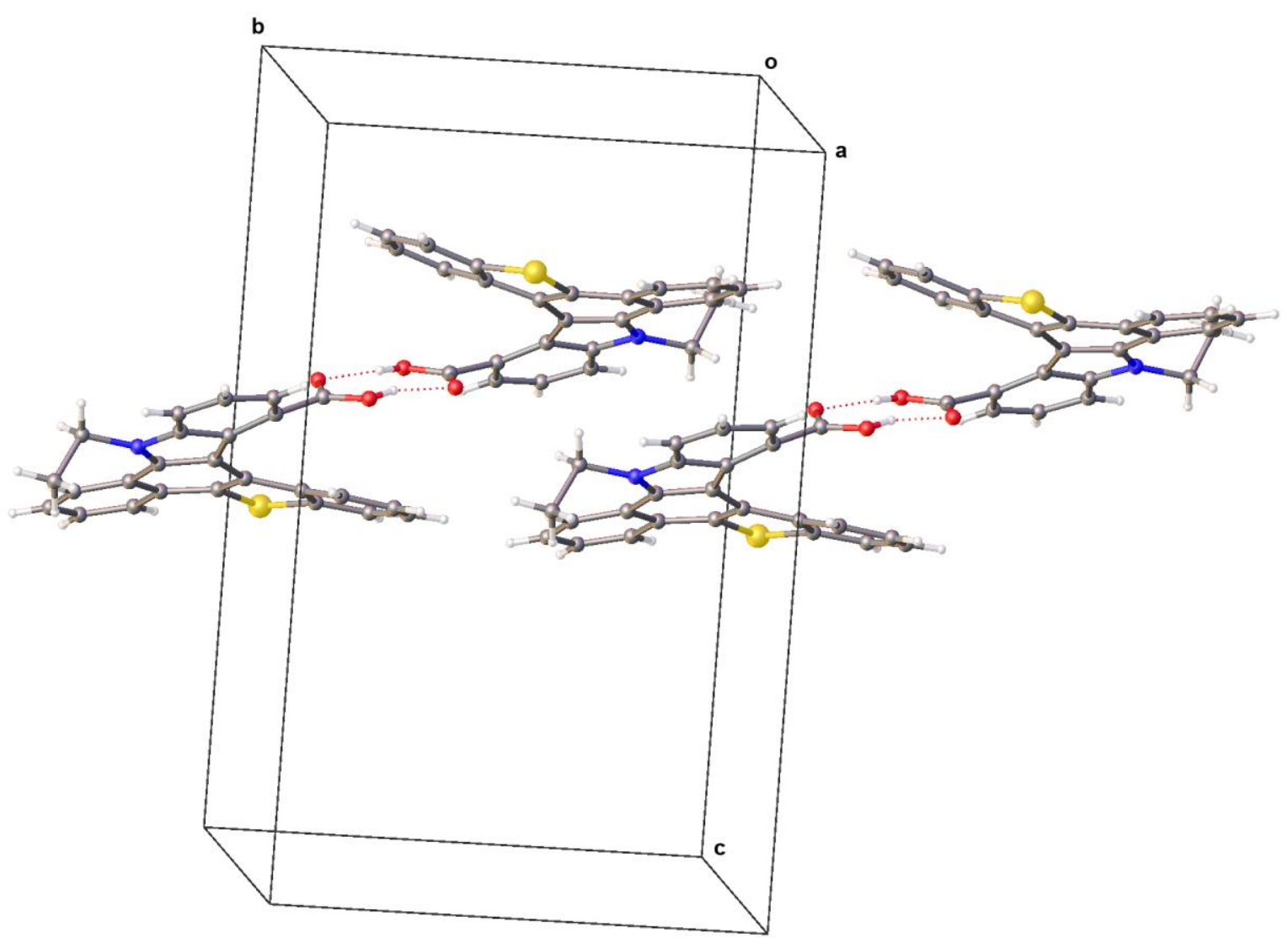

Figure S7: Packing in crystal structure of $\mathbf{1 0 d}$ 


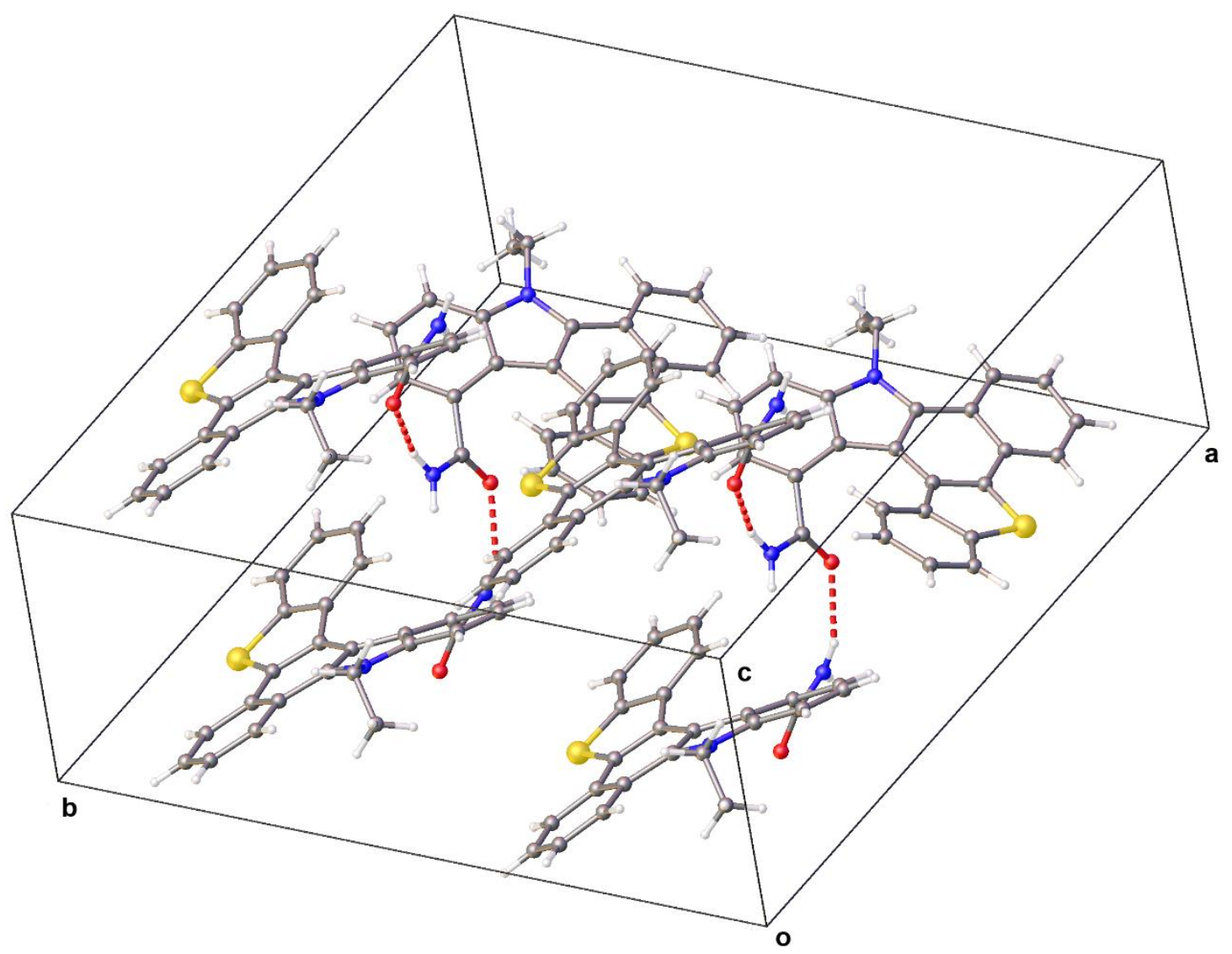

Figure S8: Packing in crystal structure of $\mathbf{1 0 e}$ 


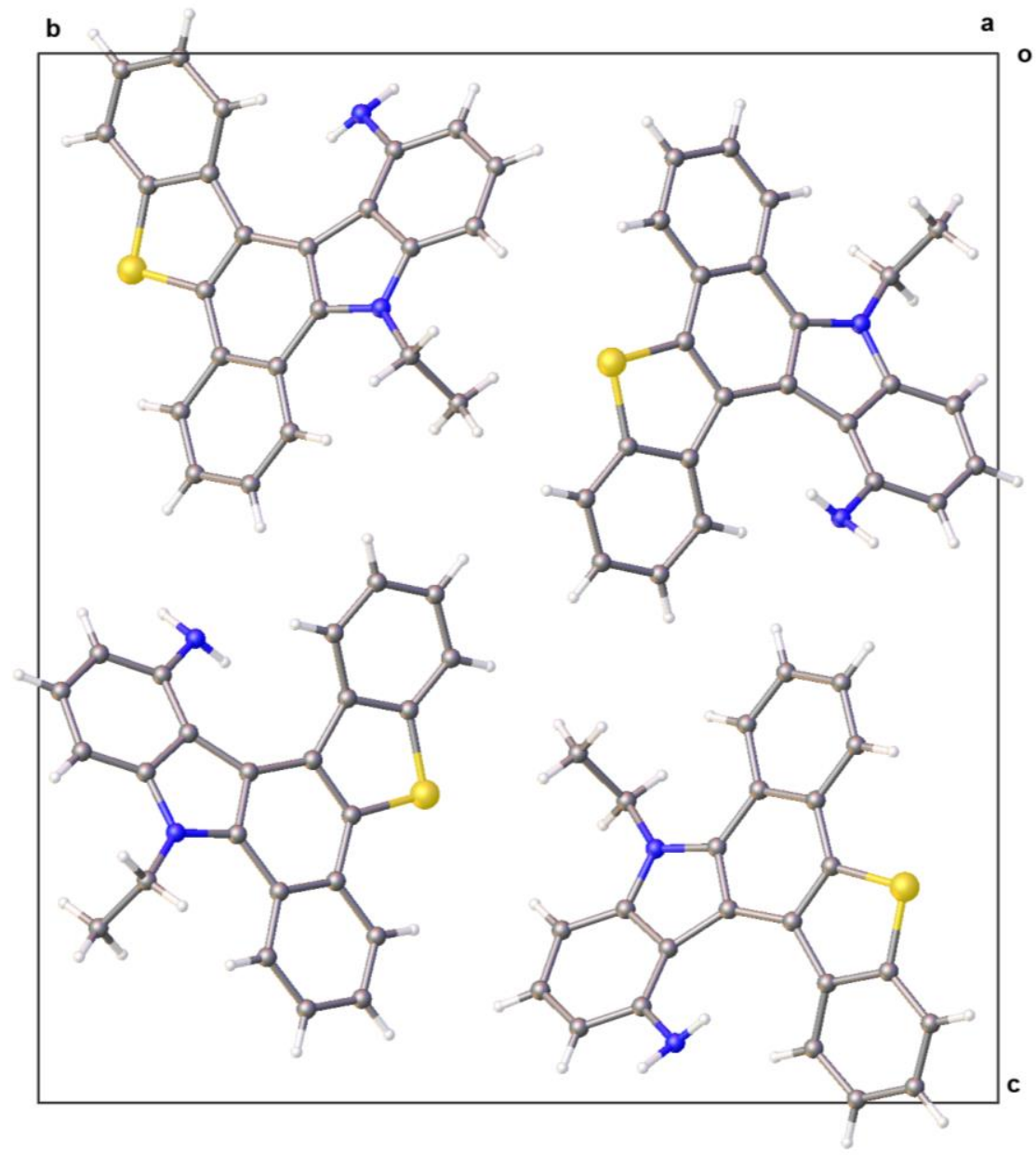

Figure S9: Packing in crystal structure of $\mathbf{1 0 f}$ 


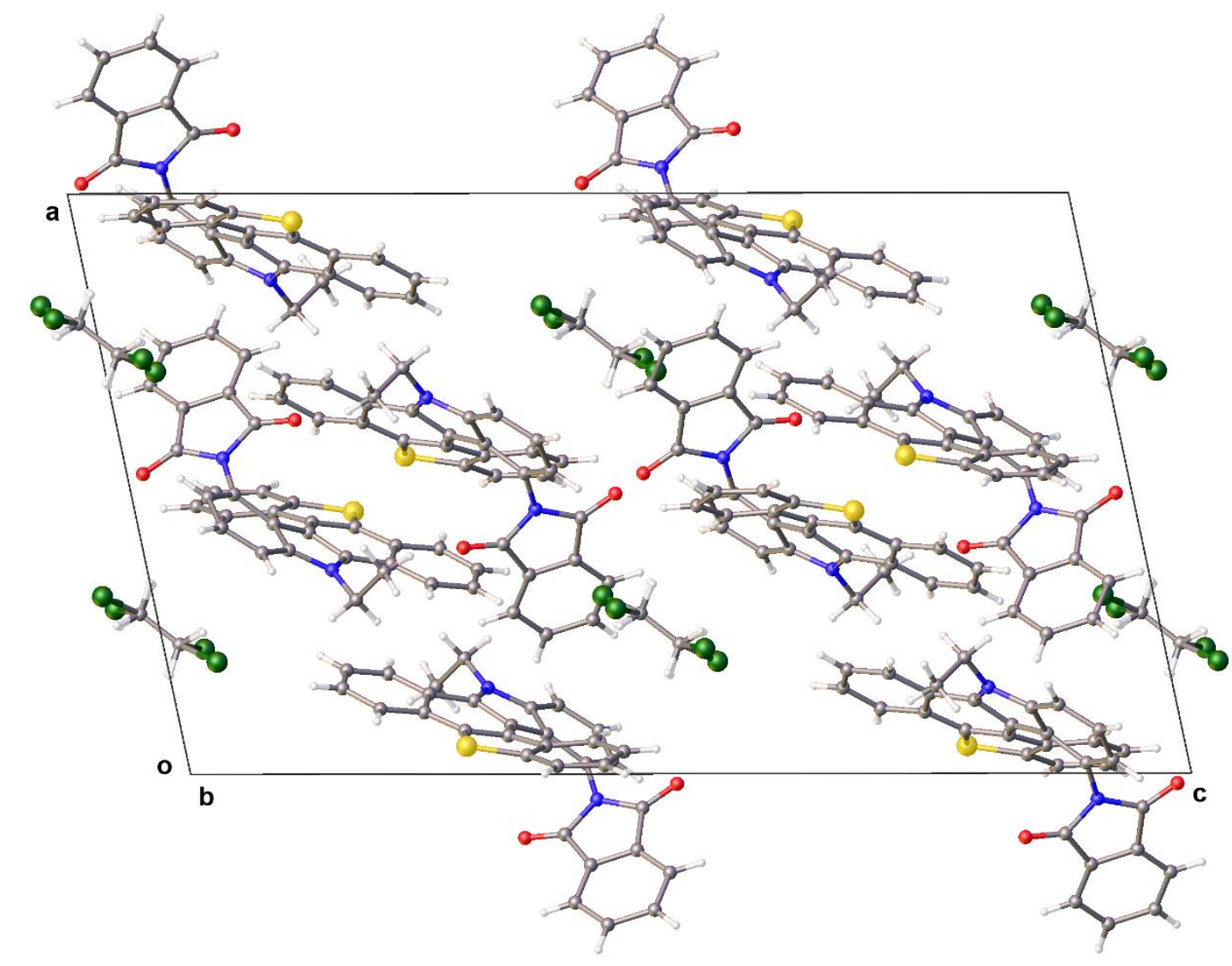

Figure S10: Packing in crystal structure of $\mathbf{1 0 g}$ 
Absorption, emission and excitation spectra
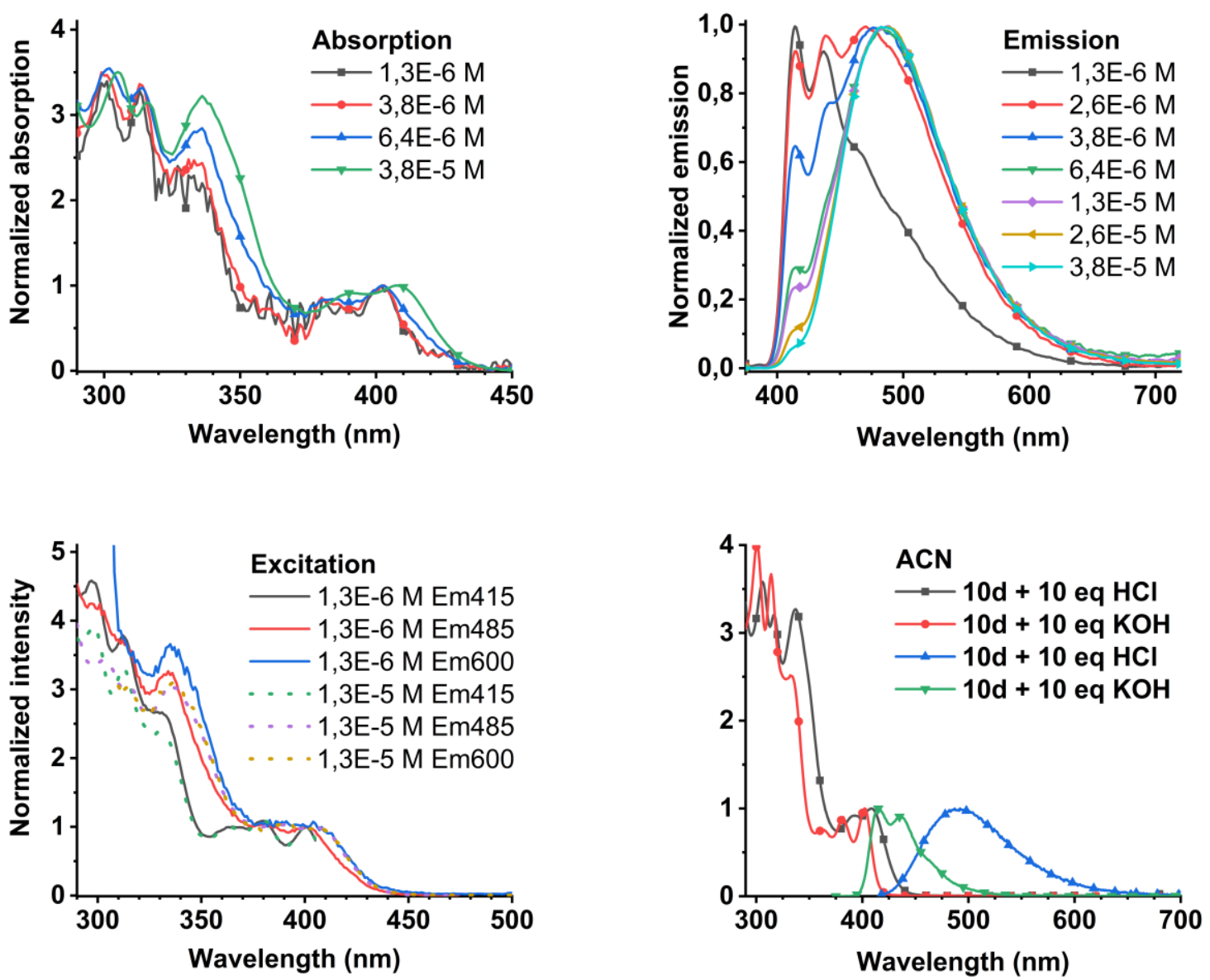

Figure S11: Absorption, emission and excitation spectra at different emission wavelengths for increasing concentration of $10 \mathrm{~d}$ in acetonitrile and absorption and emission spectra after the addition of $10 \mathrm{eq}$ of $\mathrm{HCl}$ and $\mathrm{KOH}$ to $10 \mathrm{~d}$ in acetonitrile. The absorption and excitation spectra were normalised the maximum of the first vibronic band and the emission spectra were normalised at the maximum. The emission spectra were recorded for an excitation wavelength of $365 \mathrm{~nm}$. 

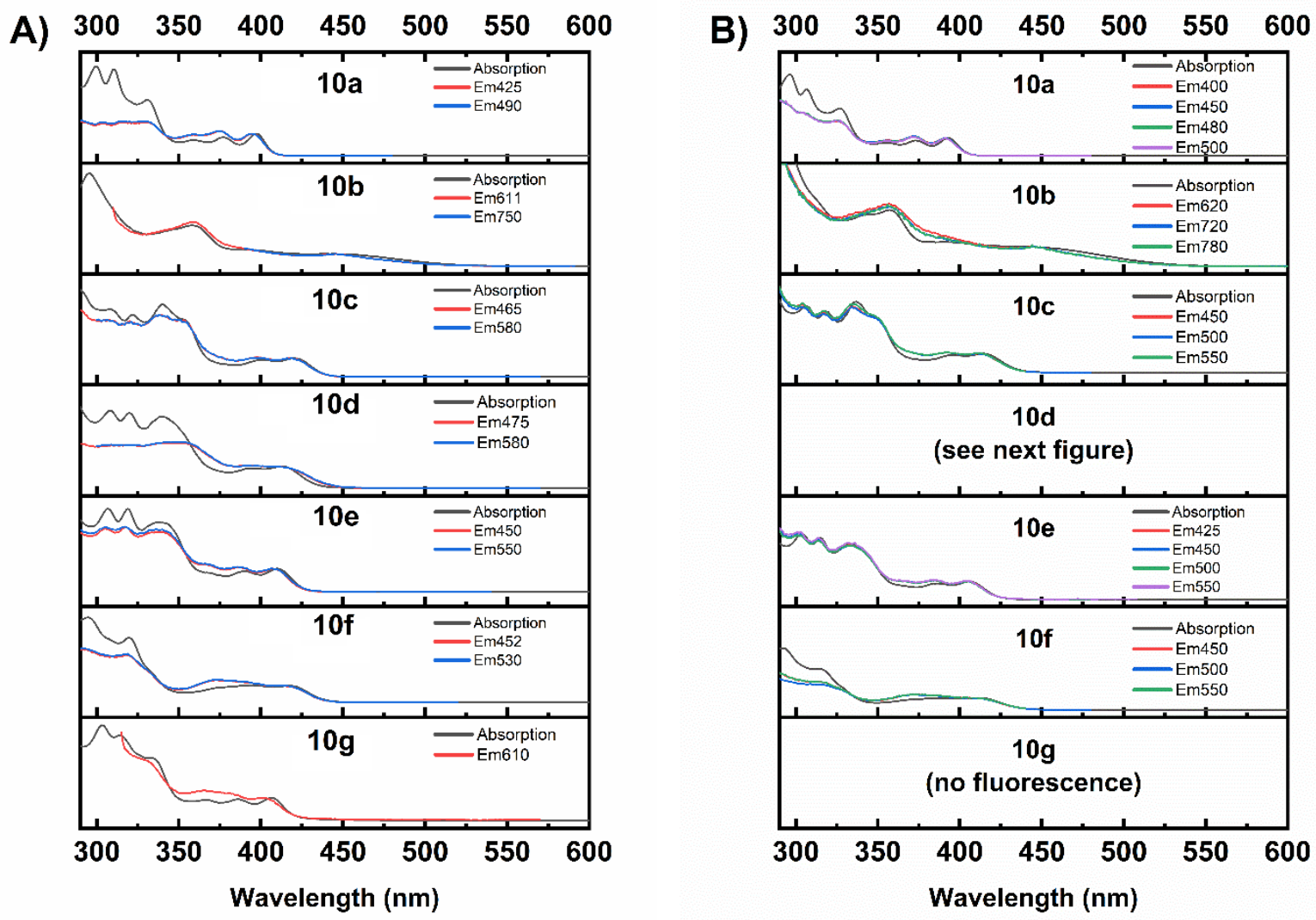

Figure S12: Excitation spectra at different observation wavelengths and absorption spectrum normalised at the first maximum. Absorption and normalised excitation spectra in toluene $(\mathbf{A})$ and acetonitrile $(\mathbf{B})$ in dilute solution at different emission wavelengths. All spectra are normalised at the maximum of the first vibronic band. 


\section{NMR-Spectra}

2-(2-Bromophenyl)-1H-indole 2a: ${ }^{1} \mathrm{H}$ NMR (300 MHz, $\left.\mathrm{CDCl}_{3}\right)$
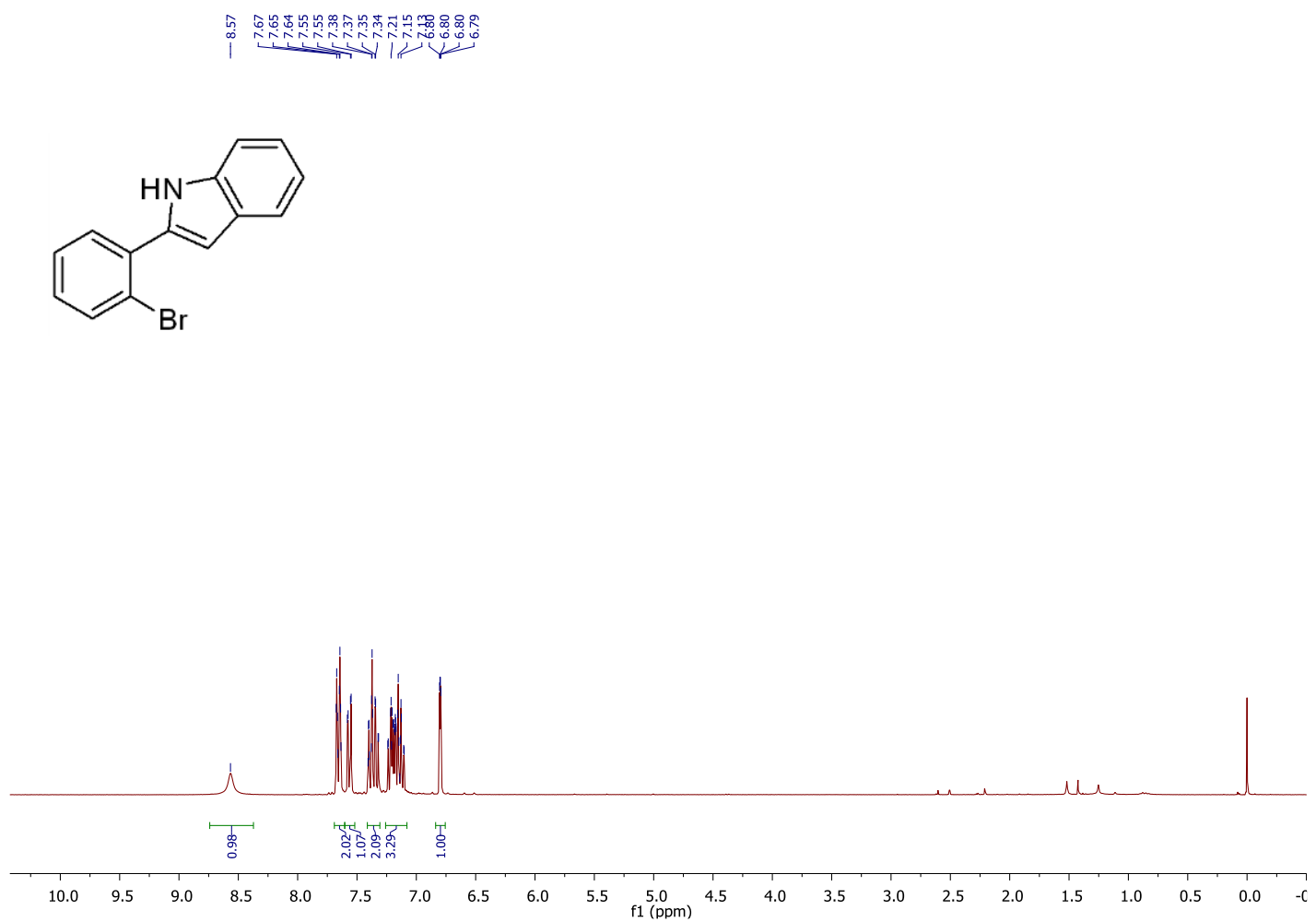
2-(2-Bromophenyl)-4-nitro-1H-indole 2b: ${ }^{1} \mathrm{H}$ NMR (400 MHz, DMSO-d6)<smiles>O=[N+]([O-])c1cccc2[nH]c(-c3ccccc3Br)cc12</smiles>
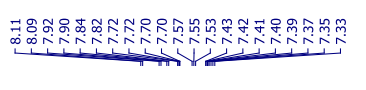

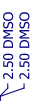

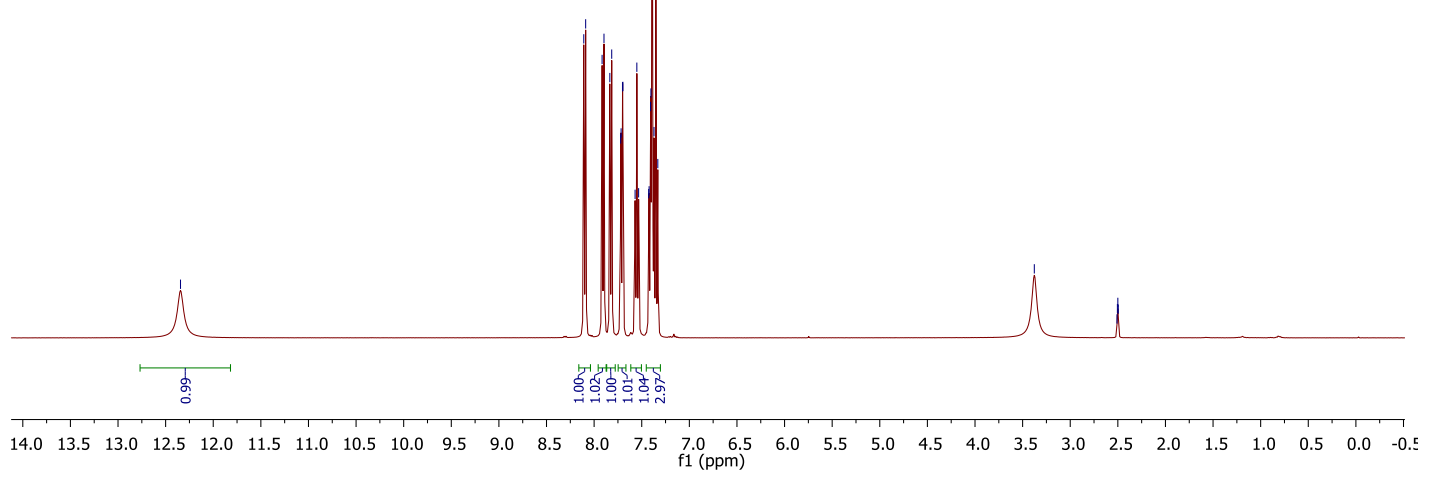

2-(2-Bromophenyl)-4-nitro-1H-indole 2b: ${ }^{13} \mathrm{C}\left\{{ }^{1} \mathrm{H}\right\}$ NMR (101 MHz, DMSO-d6)
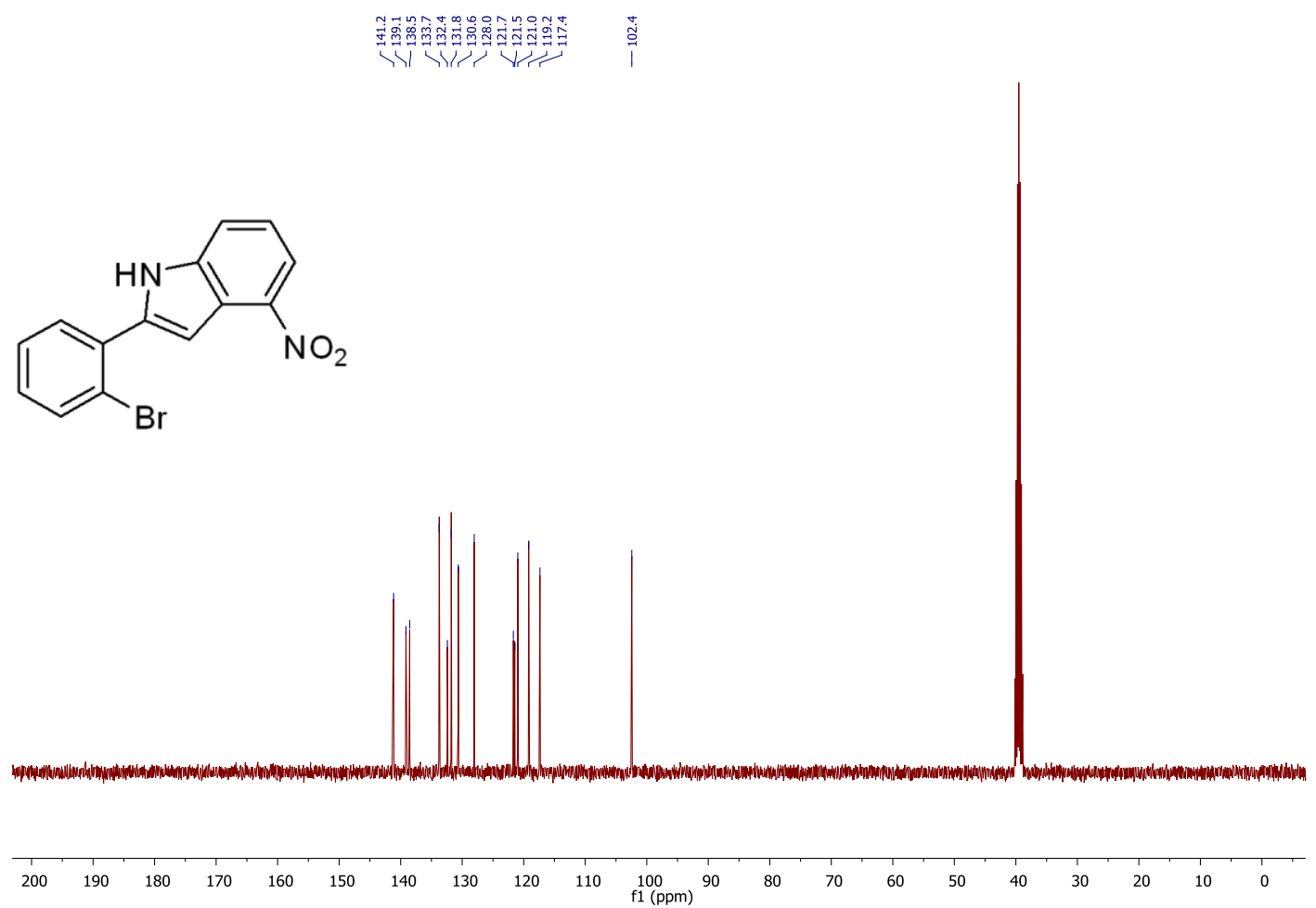
2-(2-bromophenyl)-1-ethyl-1H-indole 3a: $\left.{ }^{1} \mathrm{H} \mathrm{NMR} \mathrm{(400} \mathrm{MHz,} \mathrm{CDCl}_{3}\right)$

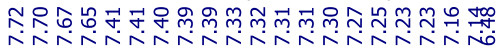<smiles>CCn1c(-c2ccccc2Br)cc2ccccc21</smiles>

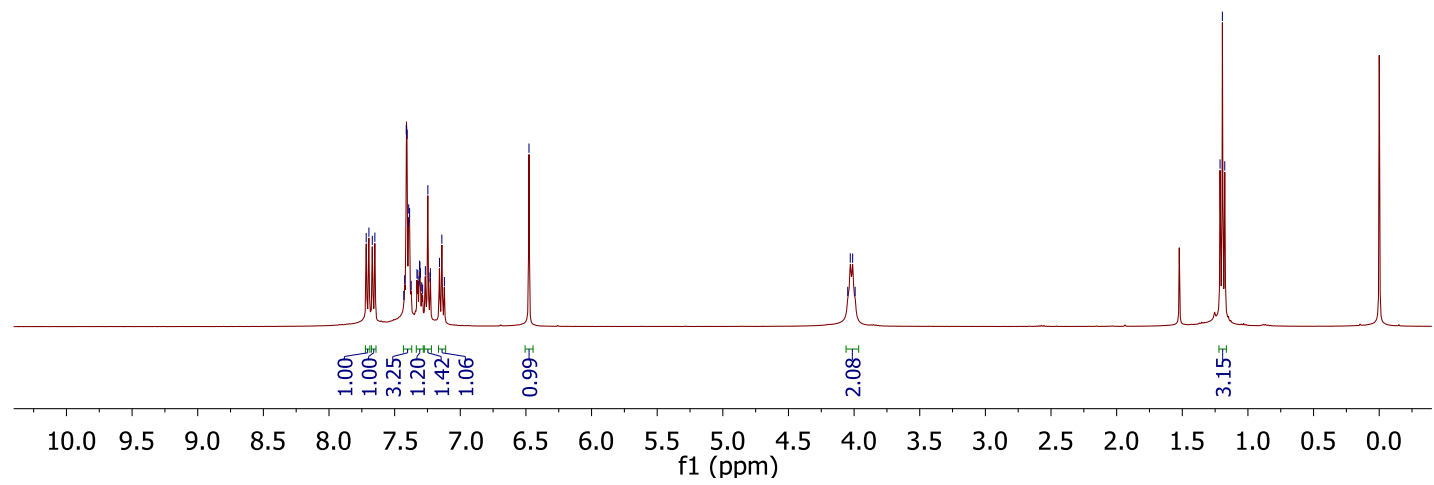

2-(2-bromophenyl)-1-ethyl-1H-indole 3a: ${ }^{13} \mathrm{C}\left\{{ }^{1} \mathrm{H}\right\}$ NMR (101 MHz, $\left.\mathrm{CDCl}_{3}\right)$

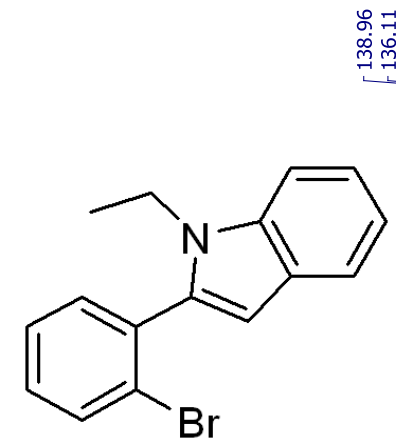

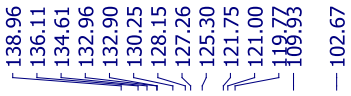

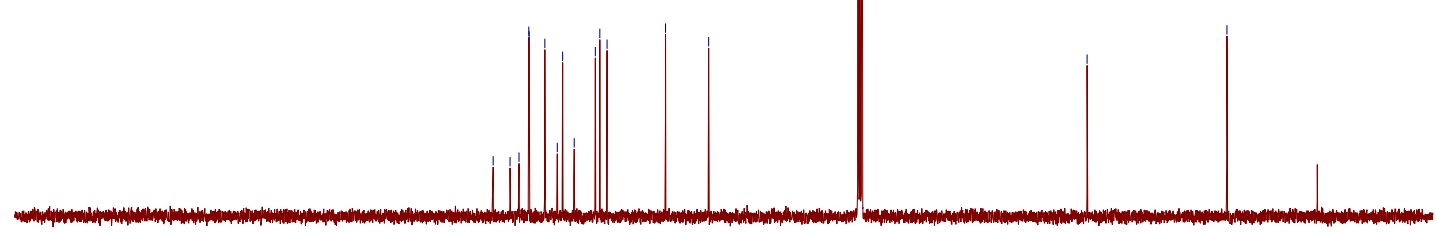

$\begin{array}{llllllllllllllllllllll}210 & 200 & 190 & 180 & 170 & 160 & 150 & 140 & 130 & 120 & 110 \quad \begin{array}{l}100 \\ \mathrm{f} 1(\mathrm{ppm})\end{array} & 90 & 80 & 70 & 60 & 50 & 40 & 30 & 20 & 10 & 0 & -10\end{array}$ 
2-(2-Bromophenyl)-1-ethyl-4-nitro-1H-indole 3b: ${ }^{1} \mathrm{H}$ NMR $\left(400 \mathrm{MHz}, \mathrm{CDCl}_{3}\right)$

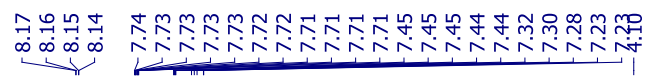<smiles>CCn1c(-c2ccccc2Br)cc2c([N+](=O)[O-])cccc21</smiles>

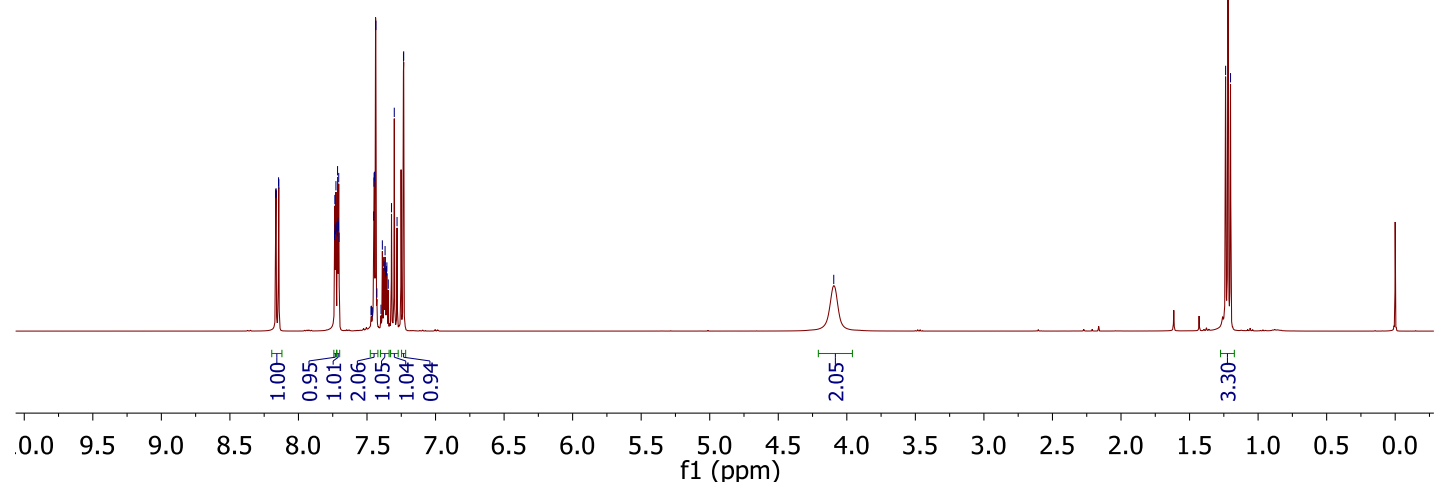

2-(2-Bromophenyl)-1-ethyl-4-nitro-1H-indole $3 \mathbf{b}:{ }^{13} \mathrm{C}\left\{{ }^{1} \mathrm{H}\right\} \mathrm{NMR}\left(101 \mathrm{MHz}, \mathrm{CDCl}_{3}\right)$

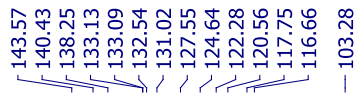<smiles>CCn1c(-c2ccccc2Br)cc2c([N+](=O)[O-])cccc21</smiles>

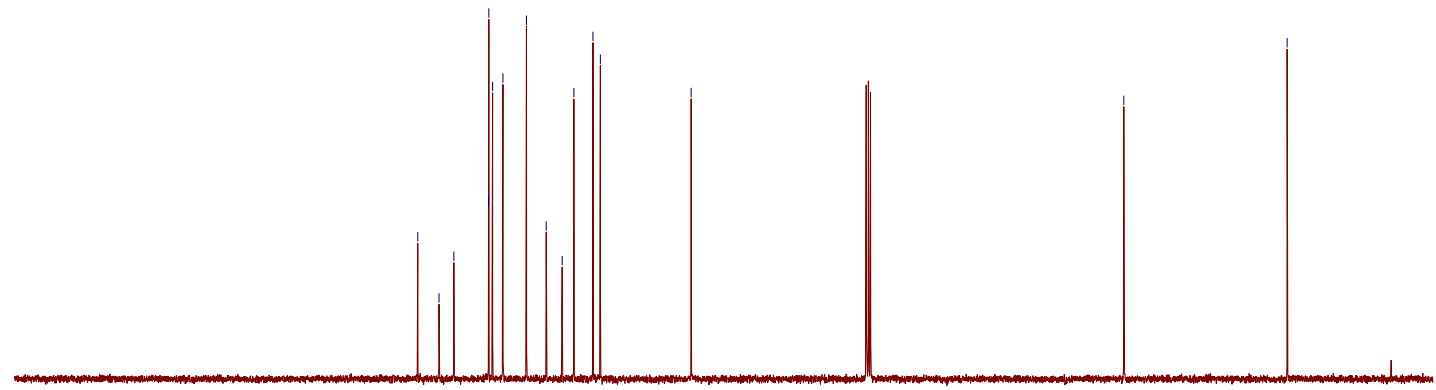


2-(2-Bromophenyl)benzo[b]thiophene 6: ${ }^{1} \mathrm{H}$ NMR (300 MHz, $\left.\mathrm{CDCl}_{3}\right)$

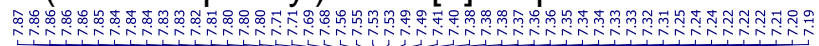<smiles>Brc1ccccc1-c1cc2ccccc2s1</smiles>

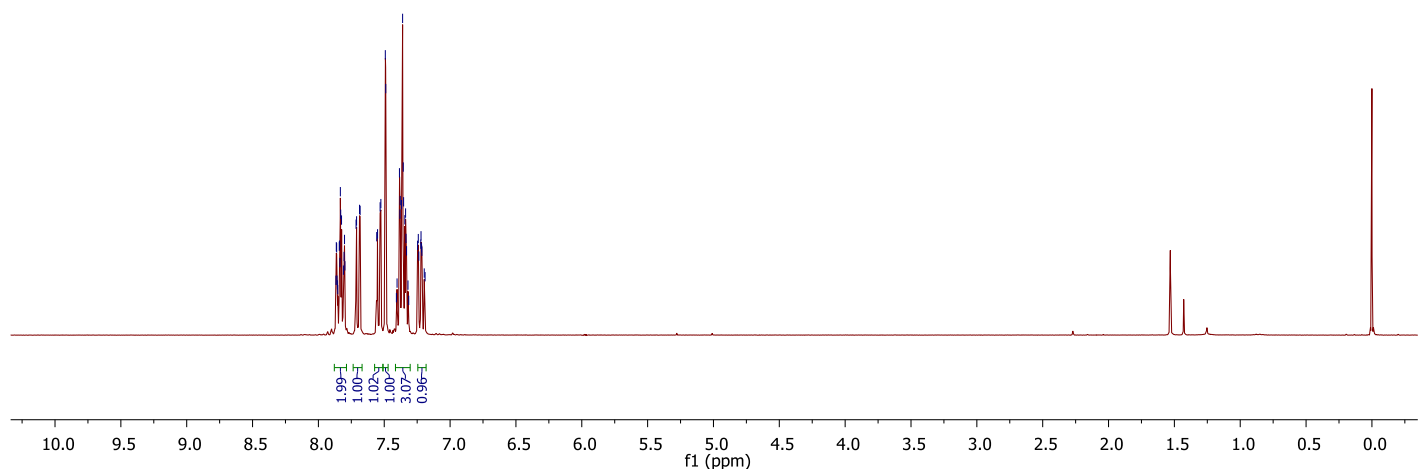


2-(2-(benzo[b]thiophen-2-yl)phenyl)-1-ethyl-1H-indole 9a: ${ }^{1} \mathrm{H} \mathrm{NMR}\left(400 \mathrm{MHz}, \mathrm{CDCl}_{3}\right)$

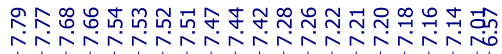<smiles>CCn1c(-c2ccccc2-c2cc3ccccc3s2)cc2ccccc21</smiles>

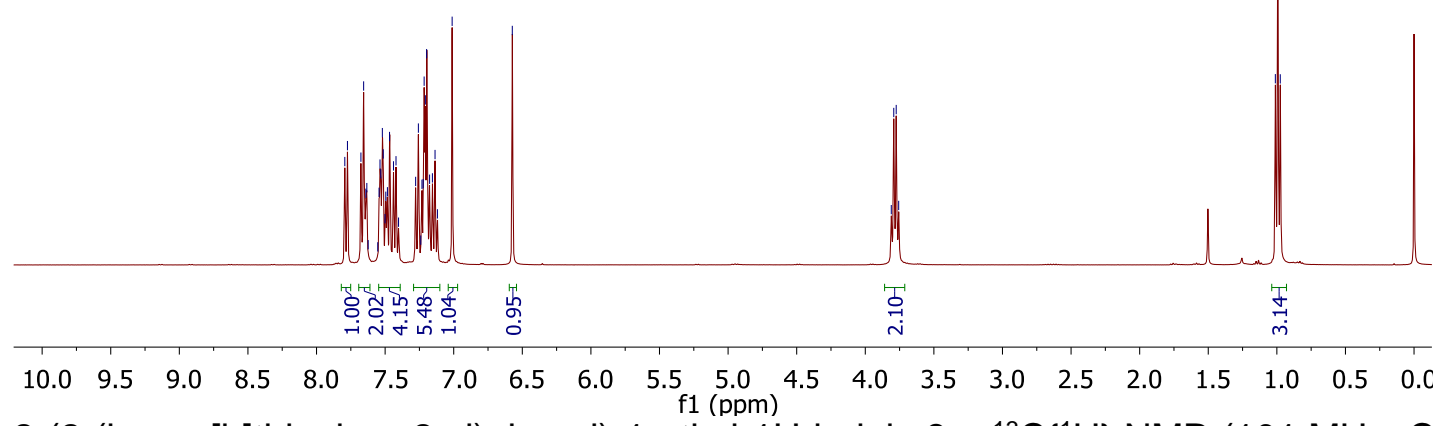

2-(2-(benzo[b]thiophen-2-yl)phenyl)-1-ethyl-1H-indole 9a: ${ }^{13} \mathrm{C}\left\{{ }^{1} \mathrm{H}\right\}$ NMR (101 $\left.\mathrm{MHz}, \mathrm{CDCl}_{3}\right)$

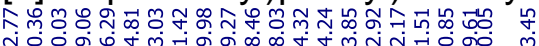

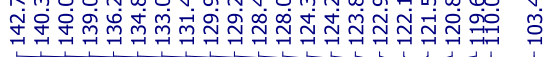<smiles>CCn1c(-c2ccccc2-c2cc3ccccc3s2)cc2ccccc21</smiles>

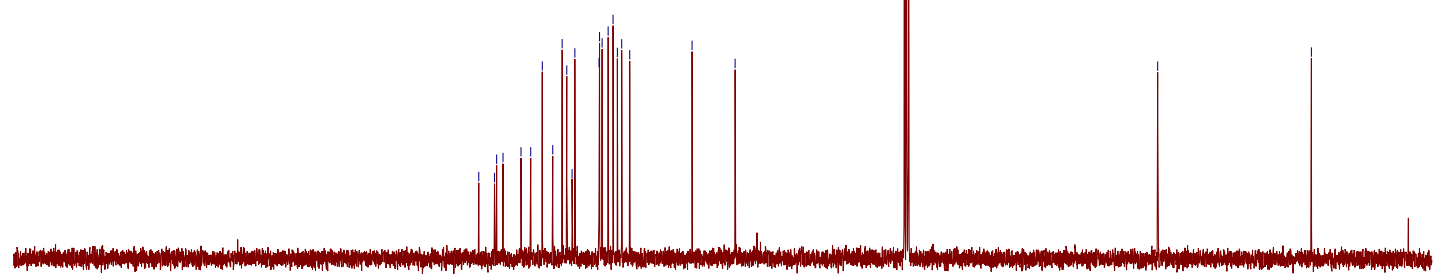

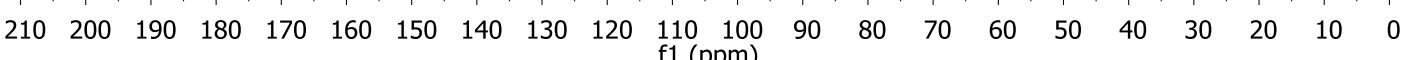


2-(2-(benzo[b]thiophen-2-yl)phenyl)-1-ethyl-4-nitro-1H-indole 9b: ${ }^{1} \mathrm{H}$ NMR $\left(400 \mathrm{MHz}, \mathrm{CDCl}_{3}\right)$

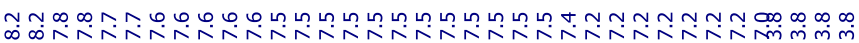<smiles>CCCn1c(-c2ccccc2-c2cc3ccccc3s2)cc2c([N+](=O)[O-])cccc21</smiles>

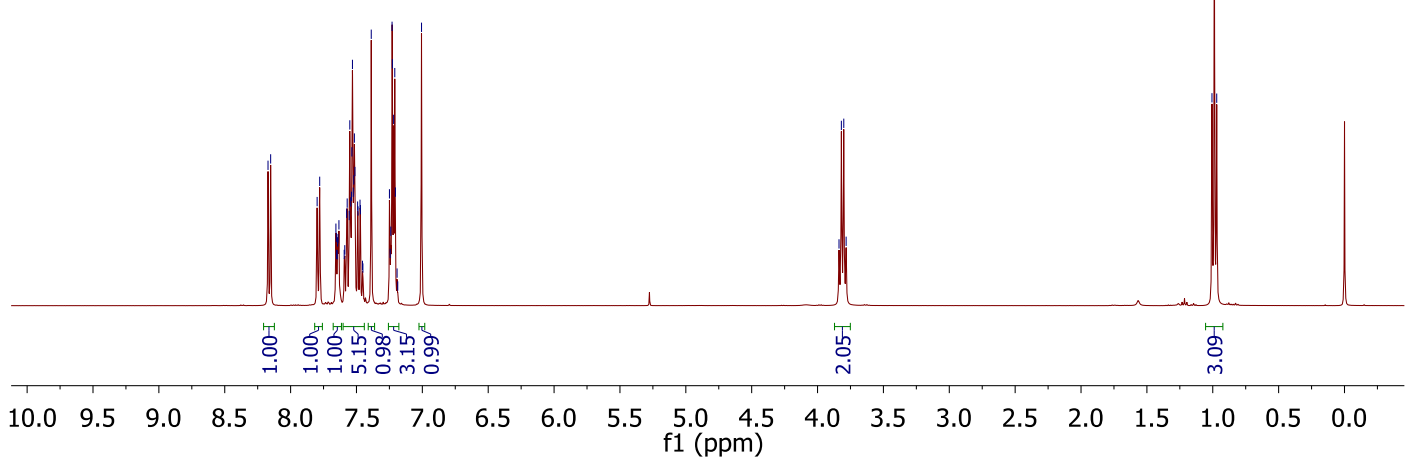

2-(2-(benzo[b]thiophen-2-yl)phenyl)-1-ethyl-4-nitro-1H-indole 9b: ${ }^{13} \mathrm{C}\left\{{ }^{1} \mathrm{H}\right\} \mathrm{NMR}(101 \mathrm{MHz}$, $\left.\mathrm{CDCl}_{3}\right)$
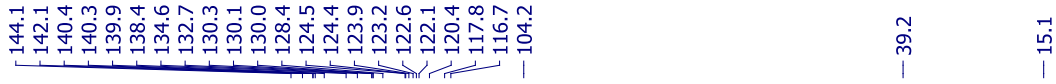<smiles>CCCn1c(-c2ccccc2-c2cc3ccccc3s2)cc2c([N+](=O)[O-])cccc21</smiles>

$\begin{array}{llllllllllllllllllllll}210 & 200 & 190 & 180 & 170 & 160 & 150 & 140 & 130 & 120 & \begin{array}{c}110 \\ \mathrm{f} 1\end{array}\left(\begin{array}{ll}100 \\ (\mathrm{ppm})\end{array}\right. & 90 & 80 & 70 & 60 & 50 & 40 & 30 & 20 & 10 & 0 & -10\end{array}$ 
2-(2-(benzo[b]thiophen-2-yl)phenyl)-1-ethyl-1 H-indole-4-carbonitrile 9c: ${ }^{1} \mathrm{H}$ NMR $(400 \mathrm{MHz}$, $\left.\mathrm{CDCl}_{3}\right)$

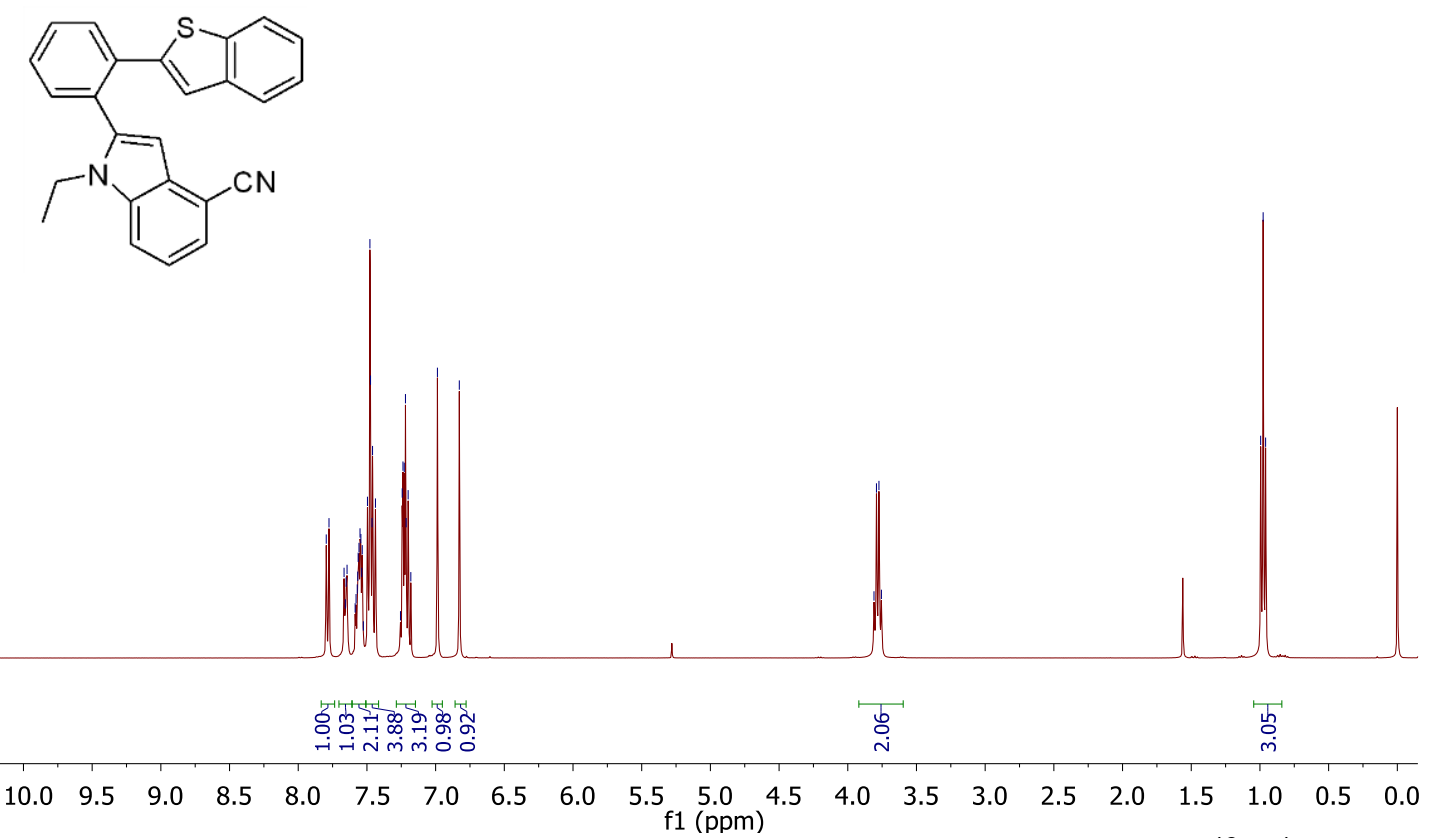

2-(2-(benzo[b]thiophen-2-yl)phenyl)-1-ethyl-1H-indole-4-carbonitrile 9c: ${ }^{13} \mathrm{C}\left\{{ }^{1} \mathrm{H}\right\} \mathrm{NMR}(101 \mathrm{MHz}$, $\left.\mathrm{CDCl}_{3}\right)$

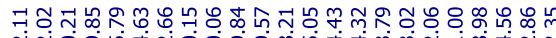

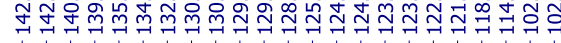<smiles>CCn1c(-c2ccccc2-c2cc3ccccc3s2)cc2c(C#N)cccc21</smiles>
. 
2-(2-(benzo[b]thiophen-2-yl)phenyl)-1-ethyl-1H-indole-4-carboxylic acid 9d: ${ }^{1} \mathrm{H} \mathrm{NMR}(400 \mathrm{MHz}$, DMSO-d6)

至

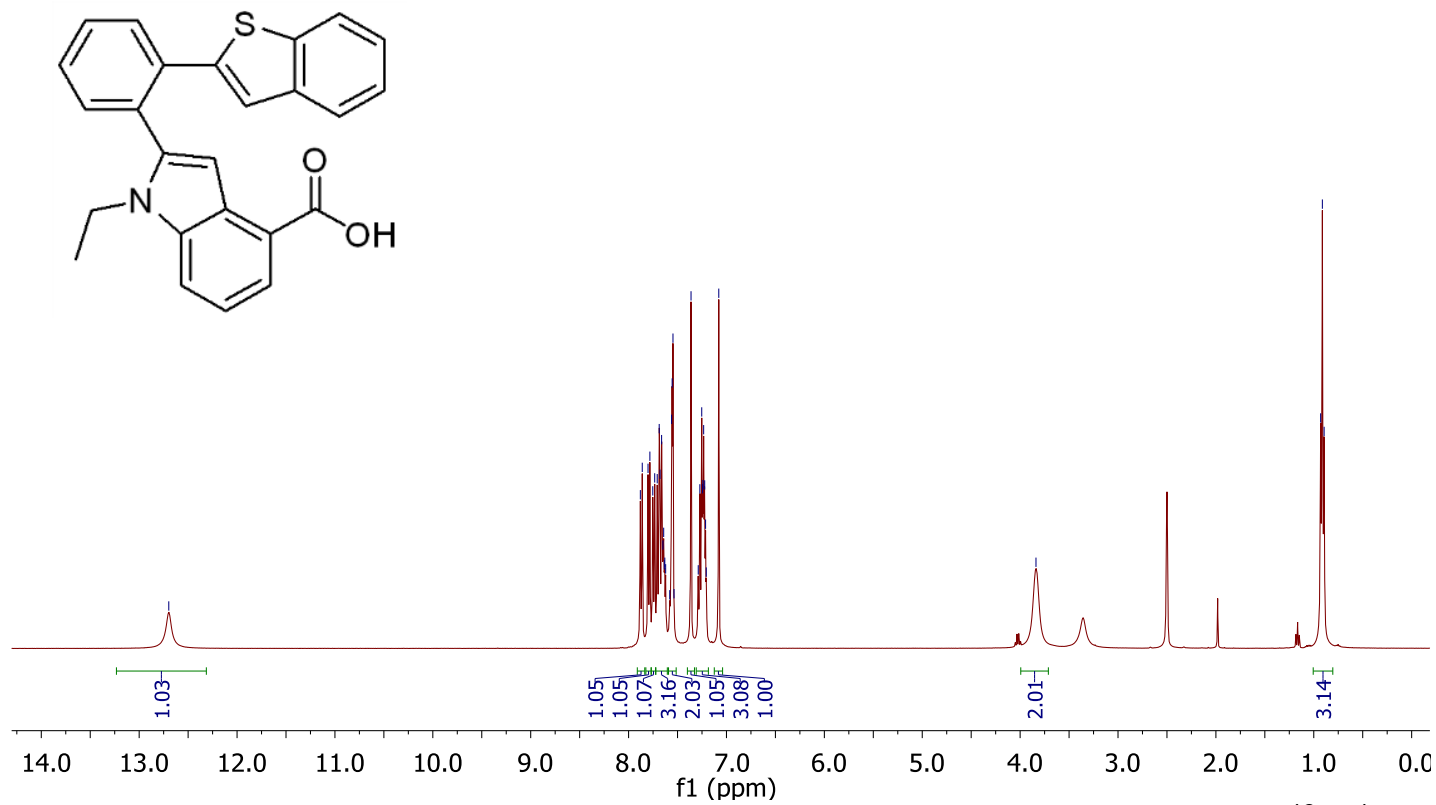

2-(2-(benzo[b]thiophen-2-yl)phenyl)-1-ethyl-1H-indole-4-carboxylic acid 9d: ${ }^{13} \mathrm{C}\left\{{ }^{1} \mathrm{H}\right\} \mathrm{NMR}(101$ $\mathrm{MHz}, \mathrm{DMSO}-\mathrm{d} 6)$

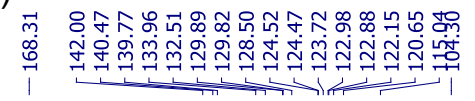<smiles>CCn1c(-c2ccccc2-c2cc3ccccc3s2)cc2c(C(=O)O)cccc21</smiles>

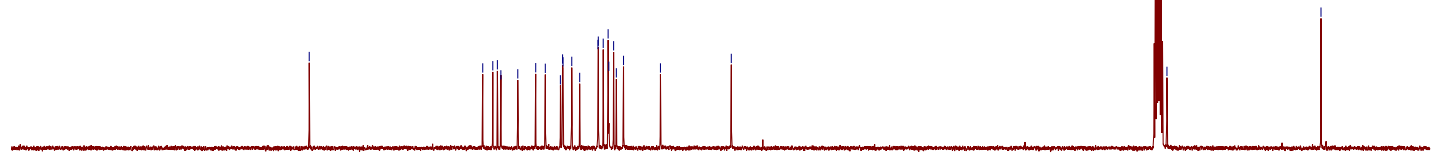

$\begin{array}{llllllllllll}210 & 200 & 190 & 180 & 170 & 160 & 150 & 140 & 130 & 120 & 110 & 100\end{array}$ f1 (ppm) 
10-ethyl-10H-benzo[a]benzo[4,5]thieno[2,3-c]carbazole 10a: ${ }^{1} \mathrm{H}$ NMR $\left(400 \mathrm{MHz}, \mathrm{CDCl}_{3}\right)$

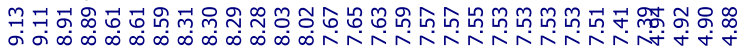
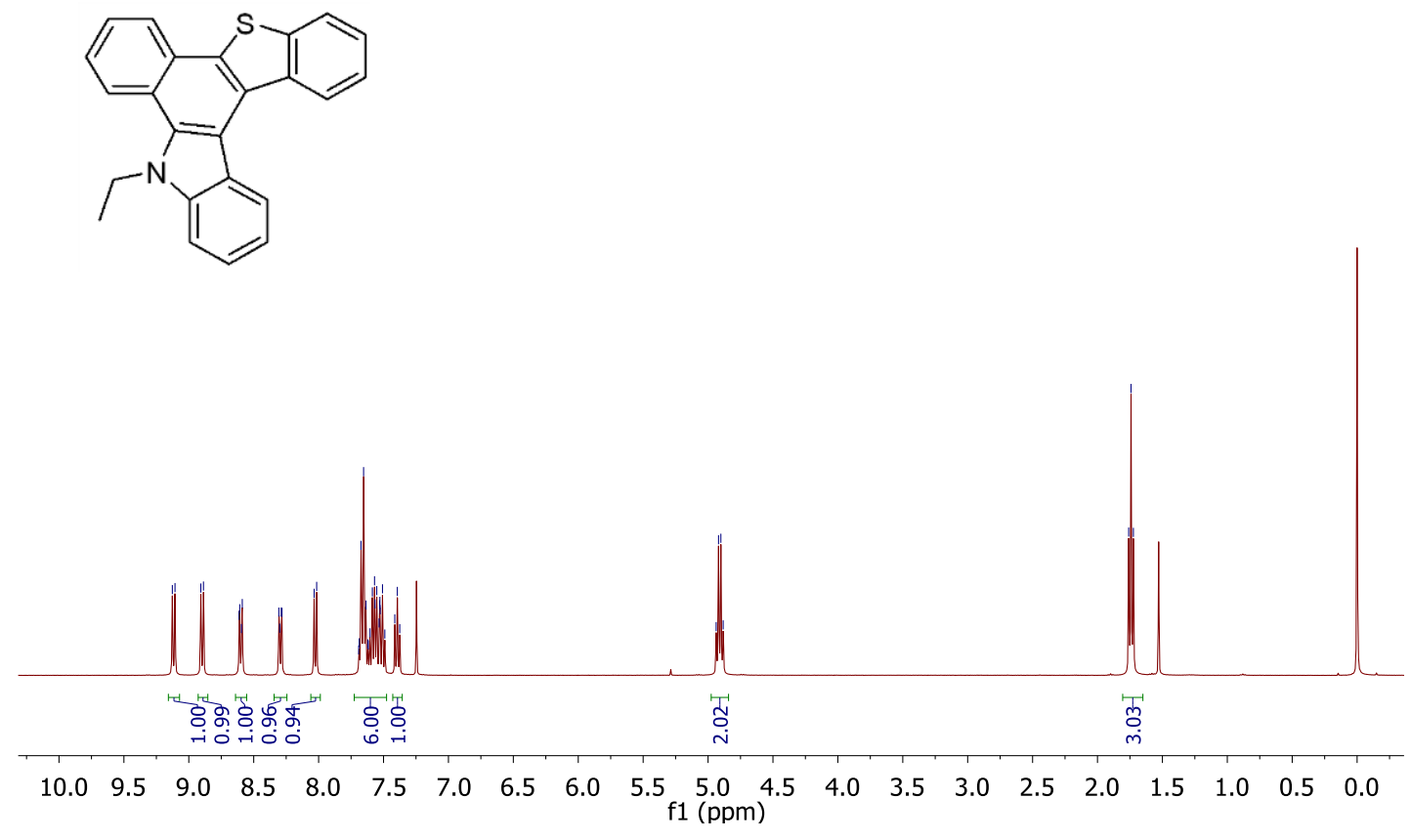

10-ethyl-10H-benzo[a]benzo[4,5]thieno[2,3-c]carbazole 10a: ${ }^{13} \mathrm{C}\left\{{ }^{1} \mathrm{H}\right\} \mathrm{NMR}\left(101 \mathrm{MHz}, \mathrm{CDCl}_{3}\right)$
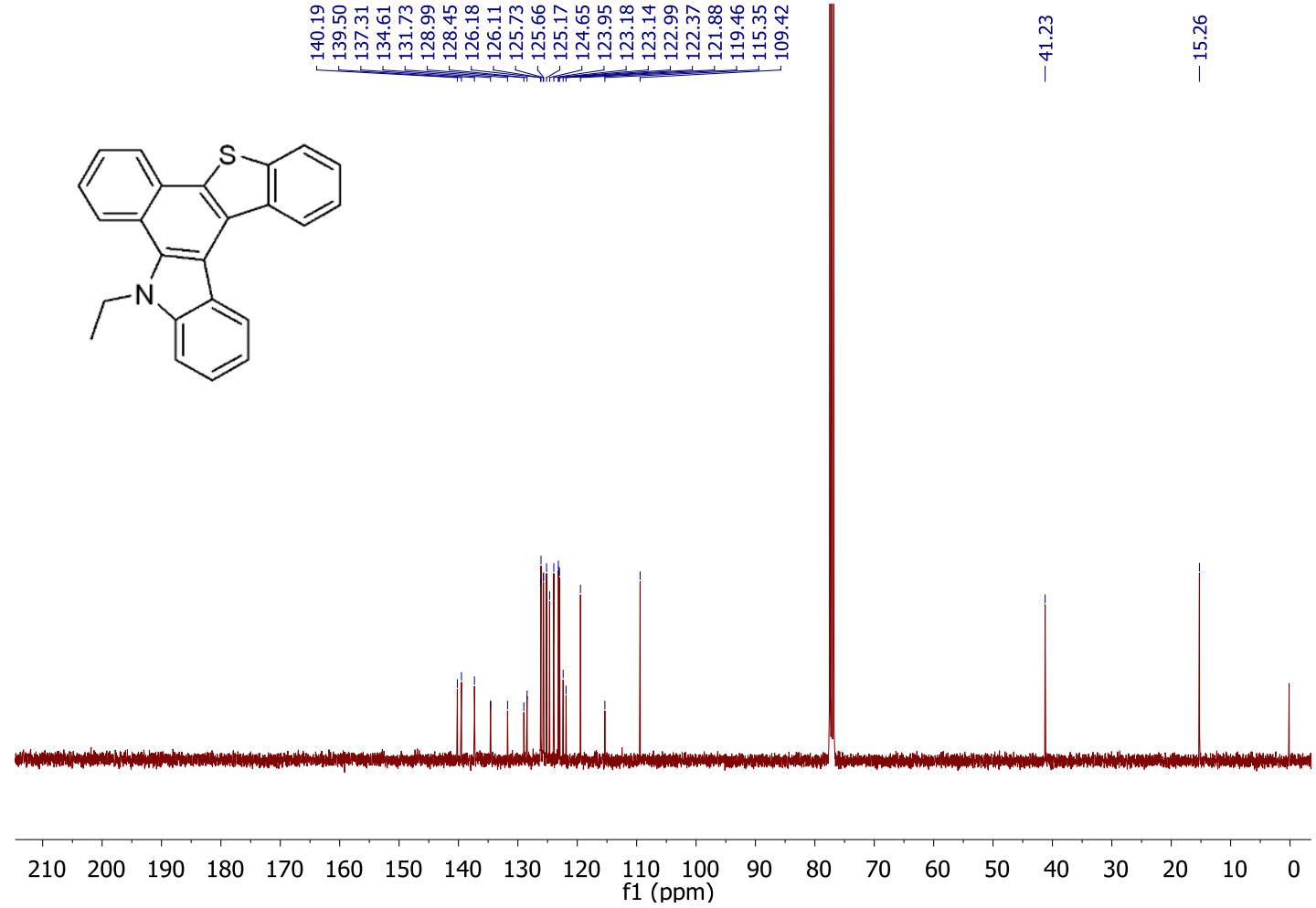
10-ethyl-14-nitro-10H-benzo[a]benzo[4,5]thieno[2,3-c]carbazole 10b: ${ }^{1} \mathrm{H} \mathrm{NMR}(400 \mathrm{MHz}$, $\left.\mathrm{CDCl}_{3}\right)$<smiles>CCn1c2ccccc2c2c3ccccc3c3c4cccc([N+](=O)[O-])c4c(c4c5ccccc5sc24)c31</smiles>

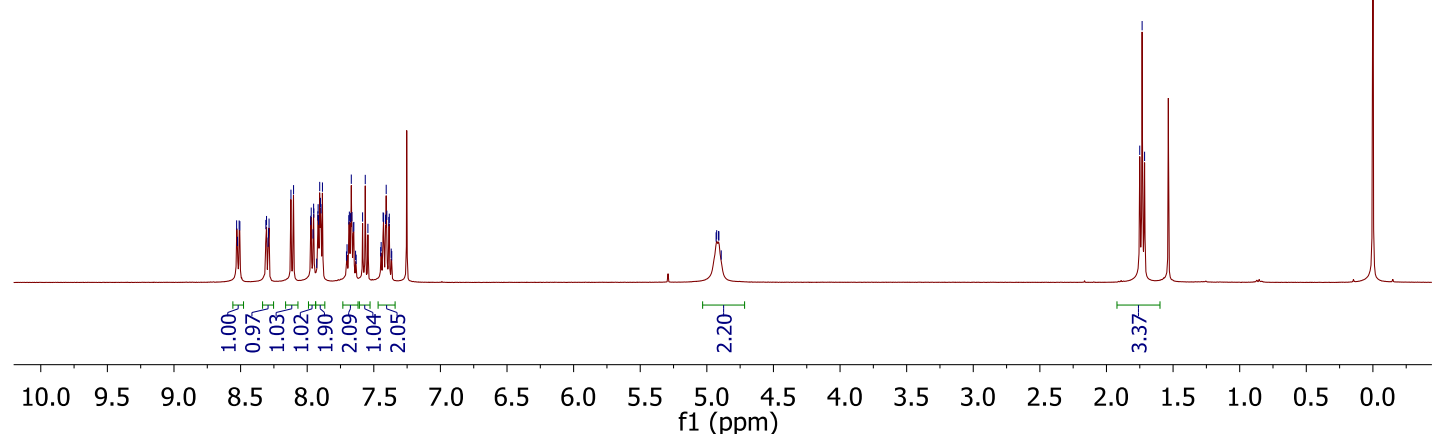

10-ethyl-14-nitro-10H-benzo[a]benzo[4,5]thieno[2,3-c]carbazole 10b: ${ }^{13} \mathrm{C}\left\{{ }^{1} \mathrm{H}\right\} \mathrm{NMR}(101 \mathrm{MHz}$, DMSO-d6)

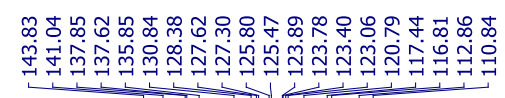<smiles>CCn1c2ccccc2c2c3ccccc3c3c4cccc([N+](=O)[O-])c4c(c4c5ccccc5sc24)c31</smiles>
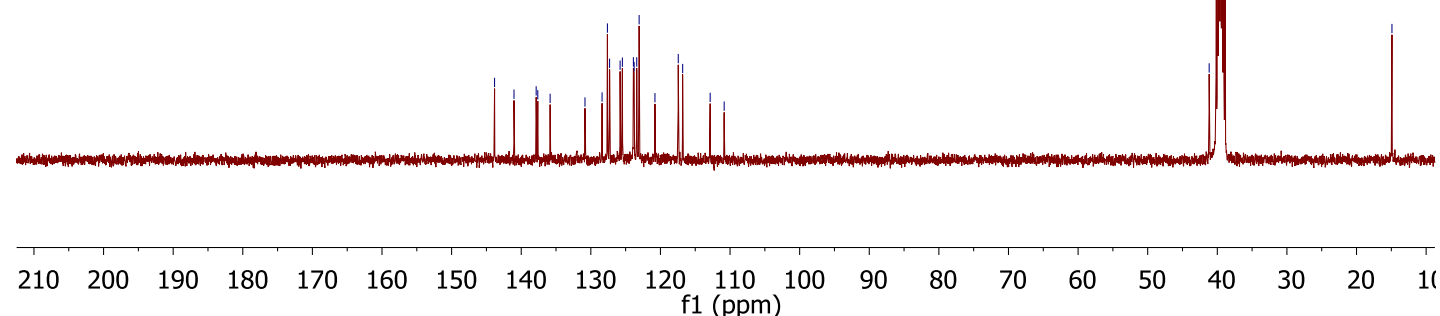
10-ethyl-10H-benzo[a]benzo[4,5]thieno[2,3-c]carbazole-14-carbonitrile 10c: ${ }^{1} \mathrm{H}$ NMR $(400 \mathrm{MHz}$, $\left.\mathrm{CDCl}_{3}\right)$

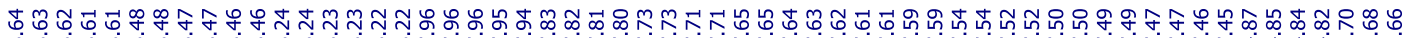

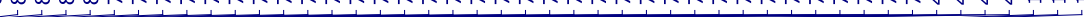<smiles></smiles>

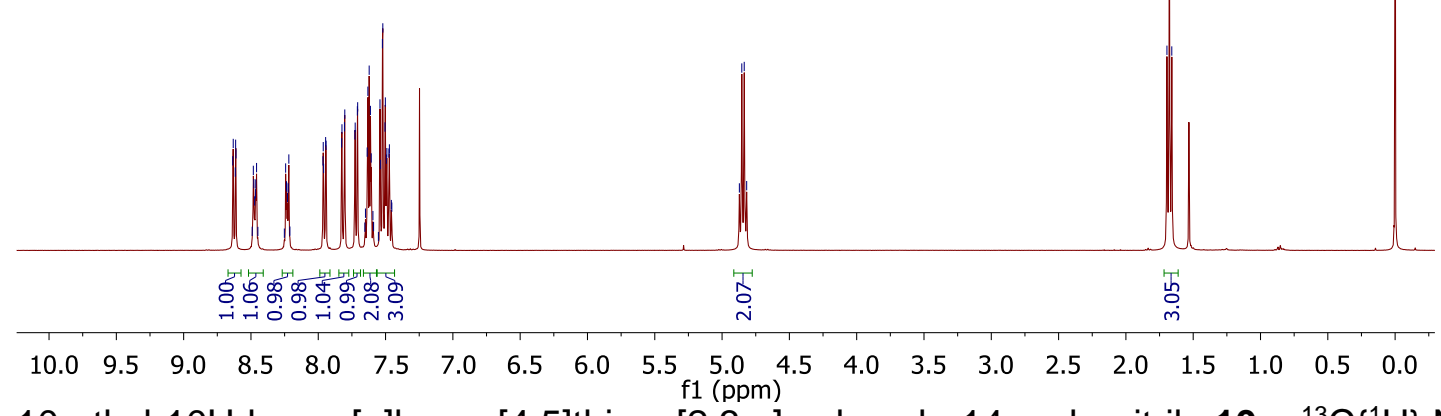

10-ethyl-10H-benzo[a]benzo[4,5]thieno[2,3-c]carbazole-14-carbonitrile $10 \mathrm{c}:{ }^{13} \mathrm{C}\left\{{ }^{1} \mathrm{H}\right\}$ NMR $(101$ $\mathrm{MHz}, \mathrm{CDCl}_{3}$ )<smiles></smiles>

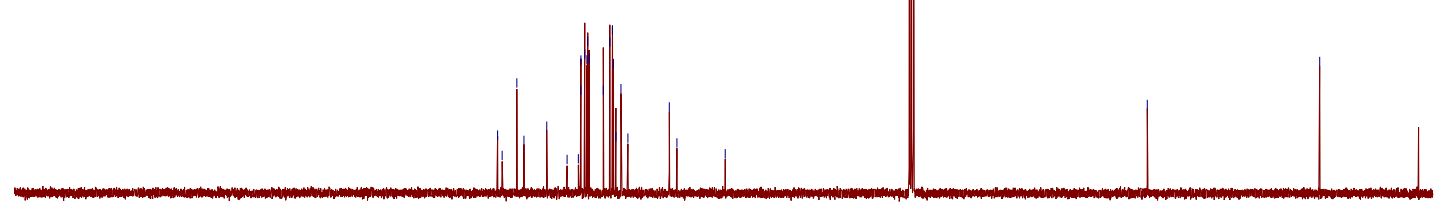

$\begin{array}{llllllllllllllllllllll}210 & 200 & 190 & 180 & 170 & 160 & 150 & 140 & 130 & 120 & \begin{array}{c}110 \\ \mathrm{f} 1(\mathrm{ppm})\end{array} & 90 & 80 & 70 & 60 & 50 & 40 & 30 & 20 & 10 & 0\end{array}$ 
10-ethyl-10H-benzo[a]benzo[4,5]thieno[2,3-c]carbazole-14-carboxylic acid 10d: ${ }^{1} \mathrm{H}$ NMR (400 $\mathrm{MHz}, \mathrm{DMSO}-\mathrm{d} 6$ )

承<smiles>CCn1c2ccccc2c2c3sc4ccccc4c3c3c(C(=O)O)cccc3c21</smiles>

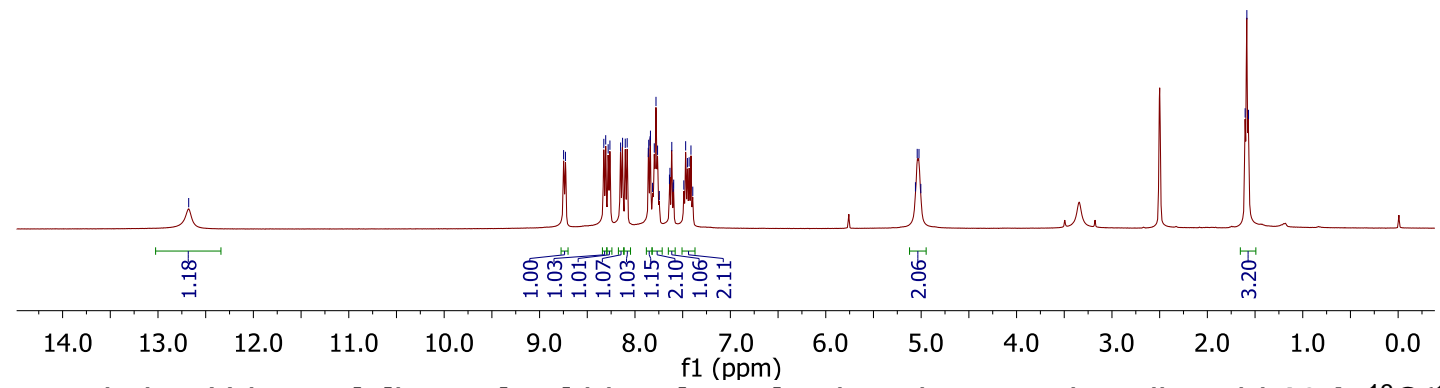

10-ethyl-10H-benzo[a]benzo[4,5]thieno[2,3-c]carbazole-14-carboxylic acid $10 \mathrm{~d}:{ }^{13} \mathrm{C}\left\{{ }^{1} \mathrm{H}\right\} \mathrm{NMR}$ (101 MHz, DMSO-d6)

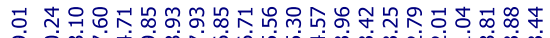

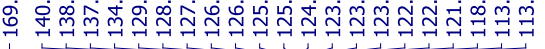<smiles></smiles>

$\begin{array}{llllllllllll}210 & 200 & 190 & 180 & 170 & 160 & 150 & 140 & 130 & 120 & \begin{array}{l}110 \\ \mathrm{f} 1(\mathrm{ppm})\end{array}\end{array}$ 
10-ethyl-10H-benzo[a]benzo[4,5]thieno[2,3-c]carbazole-14-carboxamide $10 \mathrm{e}:{ }^{1} \mathrm{H}$ NMR (300 $\left.\mathrm{MHz}, \mathrm{CDCl}_{3}\right)$

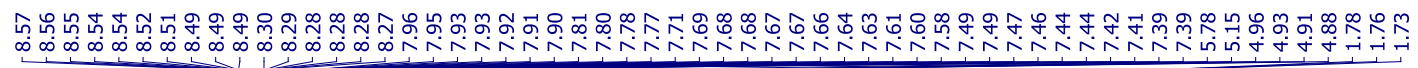

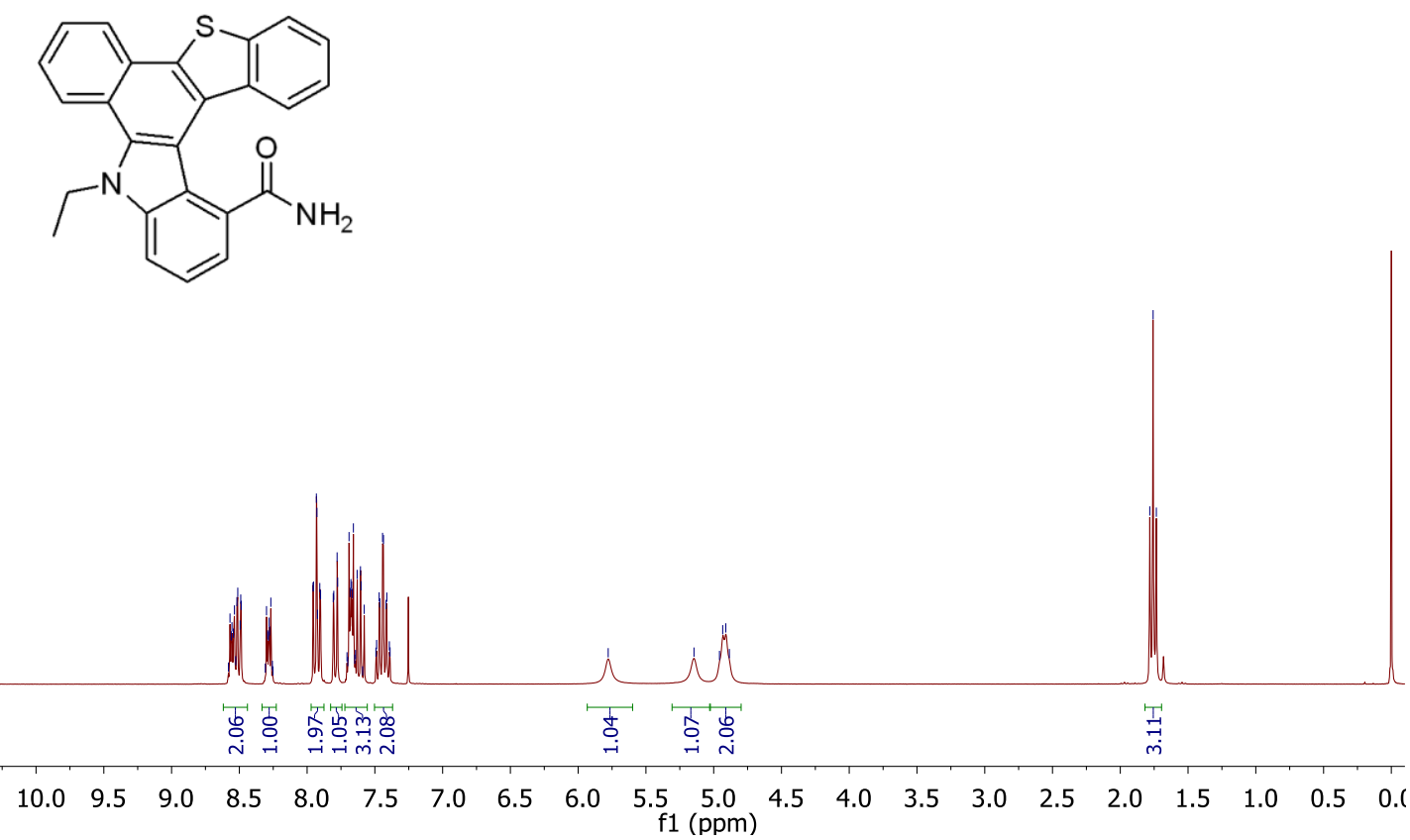

10-ethyl-10H-benzo[a]benzo[4,5]thieno[2,3-c]carbazole-14-carboxamide $10 \mathrm{e}:{ }^{13} \mathrm{C}\left\{{ }^{1} \mathrm{H}\right\}$ NMR $(75$ $\left.\mathrm{MHz}, \mathrm{CDCl}_{3}\right)$

ผ

定<smiles></smiles>

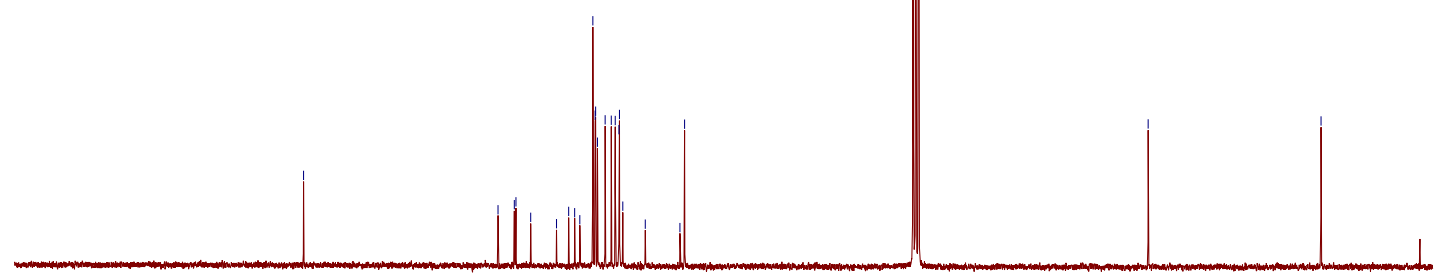

$\begin{array}{lllllllllllllllllllllll}210 & 200 & 190 & 180 & 170 & 160 & 150 & 140 & 130 & 120 & 110 & 100 & 90 & 80 & 70 & 60 & 50 & 40 & 30 & 20 & 10 & 0\end{array}$ 
10-ethyl-10H-benzo[a]benzo[4,5]thieno[2,3-c]carbazol-14-amine 10f: ${ }^{1} \mathrm{H} \mathrm{NMR}(400 \mathrm{MHz}$, $\left.\mathrm{CDCl}_{3}\right)$

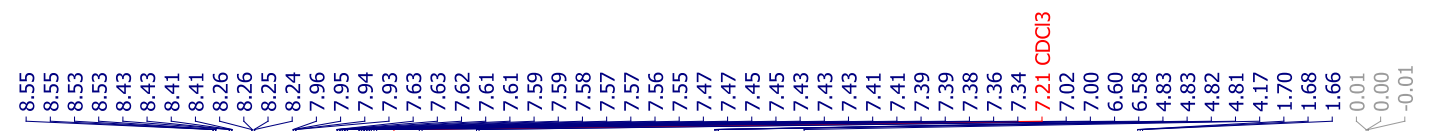<smiles></smiles>

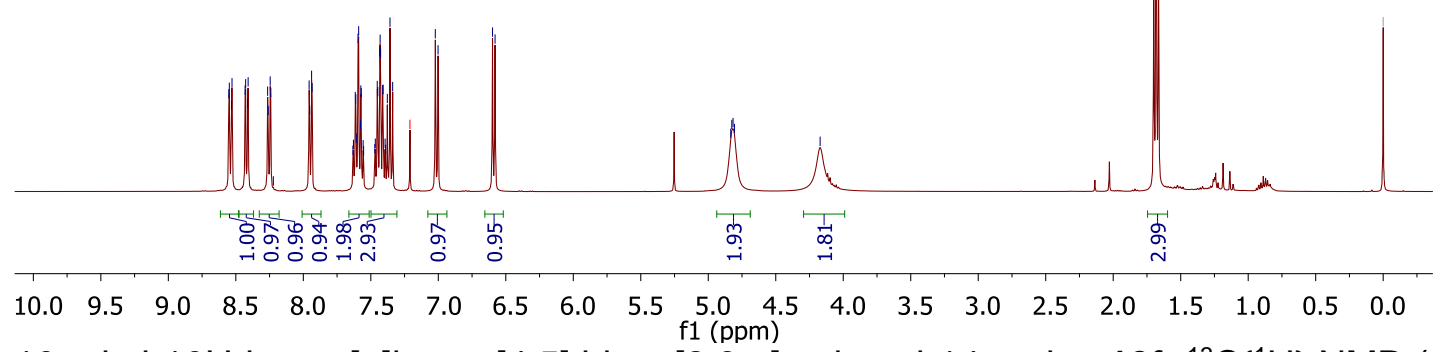

10-ethyl-10H-benzo[a]benzo[4,5]thieno[2,3-c]carbazol-14-amine 10f: ${ }^{13} \mathrm{C}\left\{{ }^{1} \mathrm{H}\right\} \mathrm{NMR}(151 \mathrm{MHz}$, $\mathrm{CDCl}_{3}$ )

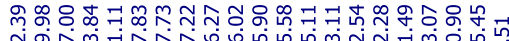

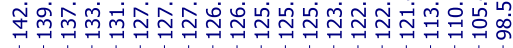

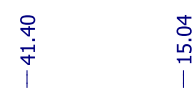<smiles></smiles>

$\begin{array}{llllllllllllllllllllll}210 & 200 & 190 & 180 & 170 & 160 & 150 & 140 & 130 & 120 & \begin{array}{c}110 \\ \mathrm{f} 1\end{array}\left(\begin{array}{ll}100 \\ (\mathrm{ppm})\end{array}\right. & 90 & 80 & 70 & 60 & 50 & 40 & 30 & 20 & 10 & 0 & -10\end{array}$ 
2-(10-ethyl-10H-benzo[a]benzo[4,5]thieno[2,3-c]carbazol-14-yl)isoindoline-1,3-dione $10 \mathrm{~g}:{ }^{1} \mathrm{H}$ NMR (300 MHz, DMSO-d6)

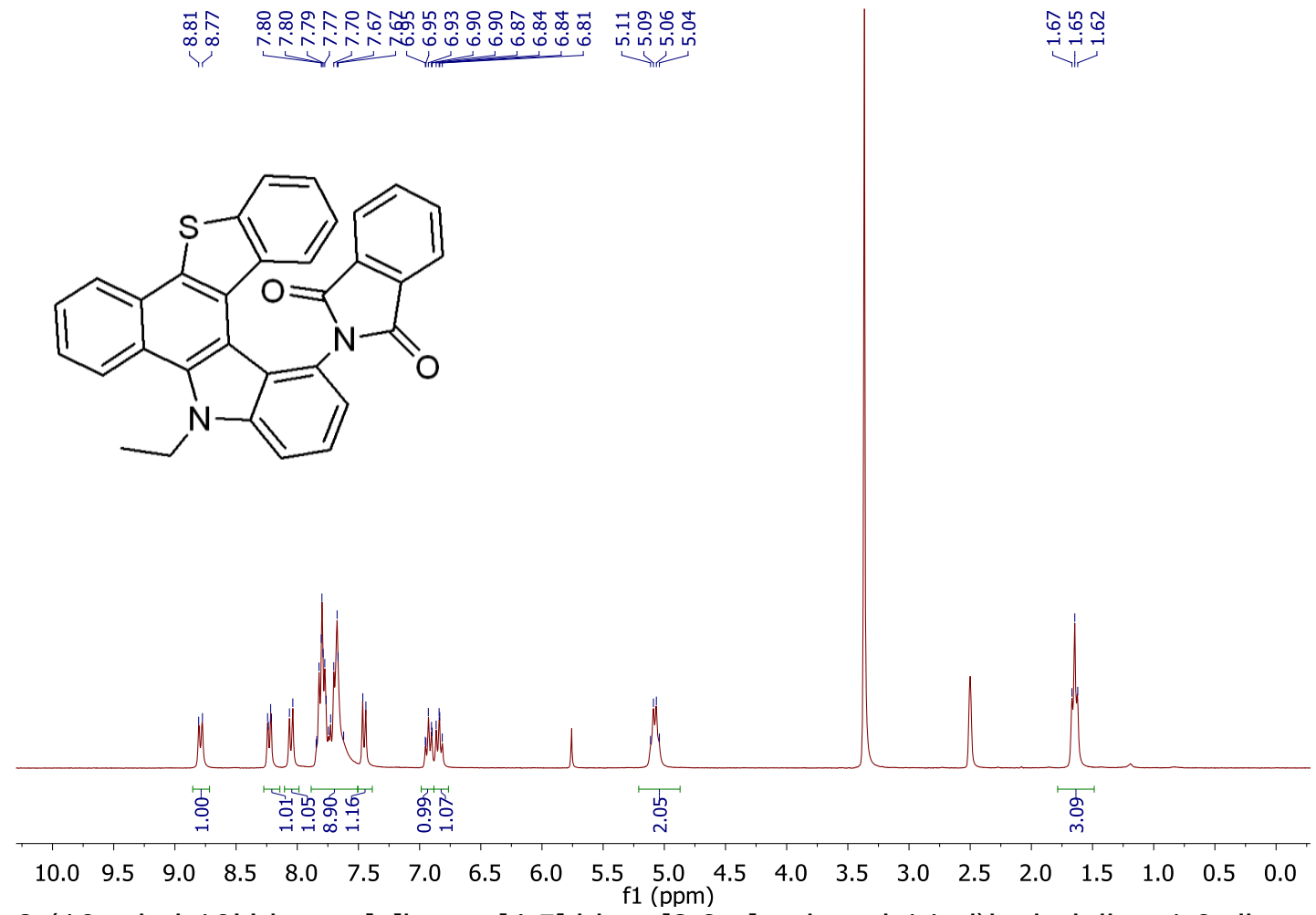

2-(10-ethyl-10H-benzo[a]benzo[4,5]thieno[2,3-c]carbazol-14-yl)isoindoline-1,3-dione 10 $\mathbf{g}$ : ${ }^{13} \mathrm{C}\left\{{ }^{1} \mathrm{H}\right\}$ NMR $(75 \mathrm{MHz}$, DMSO-d6)

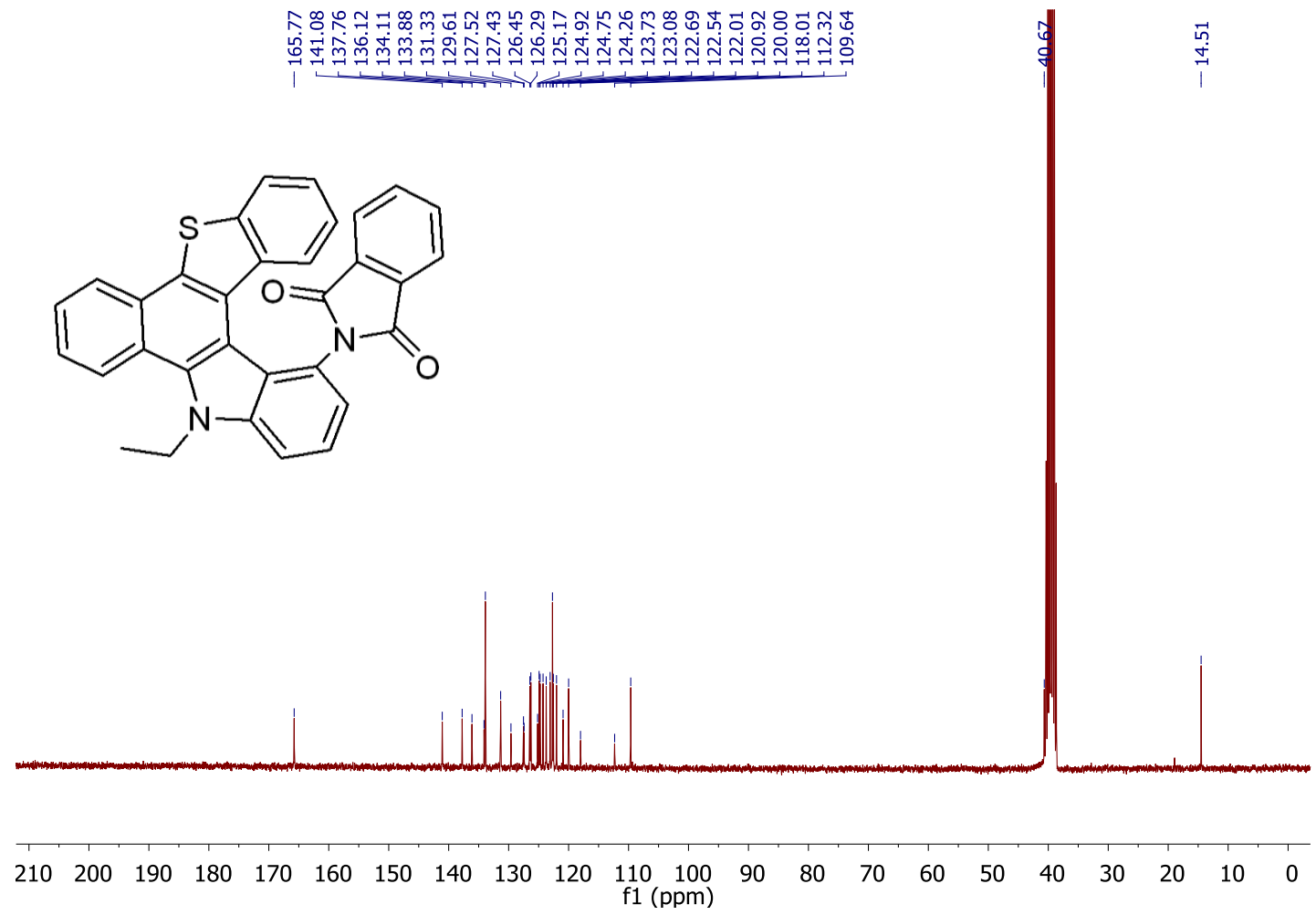

